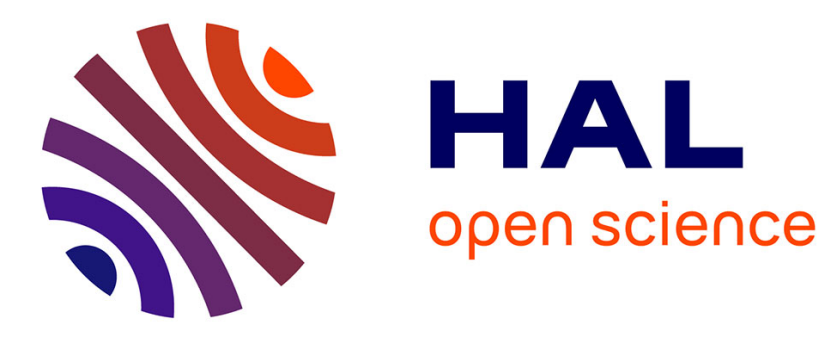

\title{
Simulating the formation of keratin filament networks by a piecewise-deterministic Markov process
}

Michael Beil, Sebastian Lück, Frank Fleischer, S. Stéphanie Portet, Wolfgang Arendt, Volker Schmidt

\section{- To cite this version:}

Michael Beil, Sebastian Lück, Frank Fleischer, S. Stéphanie Portet, Wolfgang Arendt, et al.. Simulating the formation of keratin filament networks by a piecewise-deterministic Markov process. Journal of Theoretical Biology, 2009, 256 (4), pp.518. 10.1016/j.jtbi.2008.09.044 . hal-00554513

\section{HAL Id: hal-00554513 https://hal.science/hal-00554513}

Submitted on 11 Jan 2011

HAL is a multi-disciplinary open access archive for the deposit and dissemination of scientific research documents, whether they are published or not. The documents may come from teaching and research institutions in France or abroad, or from public or private research centers.
L'archive ouverte pluridisciplinaire HAL, est destinée au dépôt et à la diffusion de documents scientifiques de niveau recherche, publiés ou non, émanant des établissements d'enseignement et de recherche français ou étrangers, des laboratoires publics ou privés. 


\section{Author's Accepted Manuscript}

Simulating the formation of keratin filament networks by a piecewise-deterministic Markov process

Michael Beil, Sebastian Lück, Frank Fleischer, Stéphanie Portet, Wolfgang Arendt, Volker Schmidt
PII:
S0022-5193(08)00504-3
DOI: doi:10.1016/j.jtbi.2008.09.044
Reference: YJTBI 5310

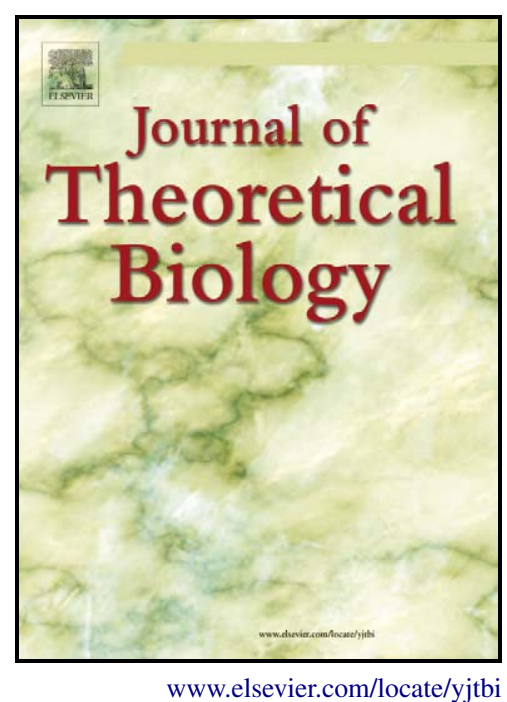

To appear in: $\quad$ Journal of Theoretical Biology

Received date: 27 February 2008

Revised date: 1 August 2008

Accepted date: $\quad 25$ September 2008

Cite this article as: Michael Beil, Sebastian Lück, Frank Fleischer, Stéphanie Portet, Wolfgang Arendt and Volker Schmidt, Simulating the formation of keratin filament networks by a piecewise-deterministic Markov process, Journal of Theoretical Biology (2008), doi:10.1016/j.jtbi.2008.09.044

This is a PDF file of an unedited manuscript that has been accepted for publication. As a service to our customers we are providing this early version of the manuscript. The manuscript will undergo copyediting, typesetting, and review of the resulting galley proof before it is published in its final citable form. Please note that during the production process errors may be discovered which could affect the content, and all legal disclaimers that apply to the journal pertain. 


\title{
Simulating the formation of keratin
}

\section{filament networks by a}

\section{piecewise-deterministic Markov process}

\author{
Michael Beil ${ }^{a * \dagger} \quad$ Sebastian Lück ${ }^{b *} \quad$ Frank Fleischer ${ }^{c}$ \\ Stéphanie Portet ${ }^{d} \quad$ Wolfgang Arendt ${ }^{e} \quad$ Volker Schmidt $^{b}$
}

${ }^{a}$ Department of Internal Medicine I, University Hospital Ulm, D-89070 Ulm, Germany, Phone: +4973150024860, Fax: +4973150044502

${ }^{b}$ Institute of Stochastics, Ulm University, D-89069 Ulm, Germany, Phone: +49 7315023531

${ }^{c}$ Medical Data Services/Biostatistics, Boehringer Ingelheim Pharma GmbH \& Co. KG, D-88397

Biberach, Germany, Phone: +497351 542372

${ }^{d}$ Department of Mathematics, University of Manitoba, Winnipeg, Manitoba, Canada, R3T 2N2,

Phone: +1 2044746933

${ }^{e}$ Institute of Applied Analysis, Ulm University, D-89069 Ulm, Germany, Phone: +49 7315023619

E-mail: michael.beil@uni-ulm.de

Keratin intermediate filament networks are part of the cytoskeleton in epithelial cells. They were found to regulate viscoelastic properties and motility of cancer cells. Due to unique biochemical properties of keratin polymers, the knowledge of the mechanisms controlling keratin network formation is incomplete. A combination of deterministic and stochastic modeling techniques can be a valuable source of information since they can describe known mechanisms of network evolution while reflecting the uncertainty with respect to a variety of molecular events. We applied the concept of

\footnotetext{
*These authors contributed equally.

${ }^{\dagger}$ corresponding author
} 
piecewise-deterministic Markov processes to the modeling of keratin network formation in high spatiotemporal resolution. The deterministic component describes the diffusion-driven evolution of a pool of soluble keratin filament precursors fueling various network formation processes. Instants of network formation events are determined by a stochastic point process on the time axis. A probability distribution controlled by model parameters exercises control over the frequency of different mechanisms of network formation to be triggered. Locations of the network formation events are assigned dependent on the spatial distribution of the soluble pool of filament precursors. Based on this modeling approach, simulation studies revealed that the architecture of keratin networks mostly depends on the balance between filament elongation and branching processes. The spatial distribution of network mesh size, which strongly influences the mechanical characteristics of filament networks, mostly depends on lateral annealing processes. This mechanism which is a specific feature of intermediate filament networks appears to be a major and fast regulator of cell mechanics.

Keywords: Cytoskeleton, Intermediate Filaments, Network Architecture, Network Formation, Piecewise-deterministic Markov Process

\section{Introduction}

The filament scaffold of the cytoskeleton determines the shape and biophysical properties of eukaryotic cells and, therefore, participates in the regulation of pivotal biological functions such as cell migration (Ballestrem et al., 2000). It consists of three biopolymer systems (actin filaments, microtubules, intermediate filaments - IF). Each filament system is characterized by specific biochemical and biophysical features (Wagner et al., 2007) which in combination with the architecture of the network determine the mechanical properties of the particular filament system (Heussinger and Frey, 2007). Although some steps of the assembly process for individual IF are now understood (Herrmann et al., 2007), the mechanisms governing the formation of networks still remain to be investigated (Oshima, 2007). IF assembly does not depend on ATP or GTP but is mo- 
dulated by posttranslational modifications of IF proteins, i.e. phosphorylation or glycosylation, which influence the solubility of IF oligomers (Coulombe and Omary, 2002). IF are non-polarized polymers and the addition of subunits and even filaments can occur at various locations along a preformed filament (Herrmann and Aebi, 2000; Windoffer et al. 2004). This extends the set of possibilities for IF network formation and remodeling in comparison to actin filaments and microtubules. In contrast to the other filament systems, there are only very few IF binding proteins known to regulate the network architecture. Models simulating actin filament or microtubular network formation are based on anisotropic growth patterns due to the polarity of these polymers. Thus, these models are not ideal to study the structural dynamics of IF networks.

IF monomers represent a heterogenous group of proteins with a tissue specific expression (Herrmann et al., 2007). Keratins are the IF proteins expressed in epithelial cells. Keratin filament networks were shown to be important for cellular mechanics (Coulombe and Wong, 2004; Magin et al., 2007). Their global architecture is regulated by phosphorylation and defines the viscoelastic properties of carcinoma cells at large deformations, thereby overriding the impact of the actin network (Beil et al., 2003). A model-based analysis revealed distinct changes of keratin network architecture in response to a modulation of keratin solubility by kinases in carcinoma cells (Beil et al., 2005 and 2006). Due to the non-linear relationship between the mesh size of polymer networks and the elastic shear modulus (Morse, 1998), even small alterations of cytoskeletal network architecture can significantly change the elasticity of the network and, hence, the mechanical characteristics of cellular compartments (Fleischer et al., 2007). Thus, the analysis of the regulation of keratin network architecture is essential for an understanding of cell mechanics which eventually might help to interfere with cancer cell migration. However, the currently available information on intracellular keratin networks is insufficient to understand the spatiotemporal regulation of their architecture. Although mathematical models simulating intracellular processes must frequently apply hypothetical conditions or deal with uncertainties, they can nevertheless provide important insights (Mogilner et al., 2006). A previous model of IF network synthesis focused on the intracellular distribution of filaments as modulated by external forces and did not regard particular spatial interactions between filaments, e.g. branching (Portet et al. 2003). Thus, we developed a new model which combines deterministic elements with stochastic processes to model distinct network formation events.

The basic subunit of keratin filaments is a heterodimer of a type I and type II keratin (Moll et 
al., 2008). Filaments are synthesized through formation of tetra- and larger oligomers which are still soluble and are, thus, subject to diffusion. These oligomers are eventually assembled into unit-length filaments which are the building blocks of longer filaments and networks (Kirmse et al., 2007). Thus, the build-up of keratin networks can be regarded at different scales as it is the case for many cellular processes (Mogilner et al., 2006). Our model is focussed on the level of structurally interacting filaments which is relevant for cell mechanics (Heussinger and Frey, 2007) and can be monitored by electron microscopy (Beil et al., 2005). At this level, however, the number of observable events is by at least a magnitude smaller than that at the level of biochemical events during oligomer synthesis and distribution. These events cannot be modeled as a bulk reaction within the limited space of cells. Thus, our approach models network growth as a sequence of discrete points in time, when macromolecular building blocks from a pool of soluble keratin oligomers are added to the network. These times are determined by a (continuous time) stochastic point process, whose inter-occurrence times are chosen as for the stochastic simulation algorithm for chemical reaction systems, which has been introduced by Gillespie (Gillespie 1977). At the times of network growth certain network formation events, whose exact molecular mechanisms and, hence, regulation are still unknown (Oshima 2007), are triggered according to a probability distribution which is controlled by model parameters. A variation of these parameters allows for studying the effect of particular network formation mechanisms on structural properties of the network. In addition, our model has to monitor the system at the scale of precursor molecules, i.e. diffusion of soluble oligomers, which can either be described as a set of random walks of individual molecules or as a bulk process by a partial differential equation (PDE). In this study, we assume that the number of soluble keratin subunits is always large enough to be modeled by a deterministic approach. By using this approach a wide range of subunit concentrations can be investigated through simulations. The spatial distribution of soluble oligomers as governed by diffusion eventually determines the specific locations of network formation events, thus, functionally interconnecting the two scales of the model.

The specific approach to combine two scale-dependent methods linking temporal dynamics with a spatial component for simulating keratin network formation appoints this model to the class of piecewise-deterministic Markov processes (PDMP - Davis, 1984).

This paper will first present the modeling concept followed by the description of the implemented algorithms for network simulations and analysis. These simulations were performed to investigate 
the impact of specific structure-defining events on network architecture, notably on the formation and distribution of meshes and connectivity, and to analyze the interplay between these events and diffusion. The results will show that the system is reaction-limited. Branching is found to be pivotal for modulating the mesh size and thereby the elasticity of keratin networks (Morse, 1998) and for generating structural inhomogeneities within networks, i.e. microgel patterns. This latter process was recently observed in carcinoma cells (Beil et al., 2005) and might be responsible for fine-tuning the mechanical properties of subcellular compartments as required during cell migration through a physically inhomogeneous environment.

\section{Model}

A detailed analysis of network architecture requires high spatial resolution of the simulation results. Thus, the model has been designed for small observation windows. In our previous studies, we investigated two-dimensional electron microscopy images of keratin networks taken from peripheral cytoplasmatic compartments (Beil et al., 2005 and 2006) which is pivotal for keratin network dynamics (Windoffer et al., 2004). These compartments are very thin and contain mostly a single layer of keratin filaments. Thus, network formation in this study is simulated on a planar square observation window $W=[0, l]^{2} \subset \mathbb{R}^{2}$ for some $l>0$. To avoid any bias caused by specific configurations of preexisting filament systems, our model is designed to study the de novo formation of a keratin network within this observation window. The initial concentration of soluble oligomers was estimated from the total length of the filament system observed in electron microscopy images of carcinoma cells (Beil et al., 2005 and 2006).

The model is based on a Markovian sequence of random network formation times, which are determined as by the Gillespie algorithm. Whereas, the classical Gillespie algorithm assumes a spatially homogeneous reaction system, the model in this study focusses on the spatial distribution of events, since it is crucial for network morphology. Therefore, the model complements the global reaction kinetics given by the Gillespie algorithm by a mechanism controlling the spatial distribution of locations for network growth. Since network formation is fueled by the pool of soluble filament precursors, locations for filament assembly are picked based on the spatial distribution of soluble precursors. The latter is modeled as a concentration field, which, in order to determine growth locations, is interpreted as a probability field. Apart from local consumption due to fila- 
ment assembly, the distribution of soluble precursor molecules is governed by a diffusion process proceeding between network formation events and described by a deterministic partial differential equation (PDE).

\subsection{Soluble and filamentous keratin pool}

Assembly of IF proceeds from a pool of soluble keratin tetramers. Since IF are highly insoluble in physiological buffers (Kirmse et al., 2007) we assume that no disassembly occurs. Filaments are the result of a longitudinal annealing process involving a variety of oligomeric subunits, which are formed through various stages of lateral annealing. Phosphorylation of monomers is a crucial mechanism regulating the transfer of keratin oligomers between the soluble and the filamentous pool. Whereas in a dephosphorylated state, keratins tend to assemble to filamentous structures (Strnad et al., 2002), phosphorylation induces dissolution of keratin filaments (Strnad et al., 2002; Omary et al., 2006). In the dephosophorylated configuration, keratin dimers exhibit a strong tendency to form tetramers of $\sim 45 \mathrm{~nm}$ length (Geisler et al., 1998). During filament assembly, lateral aggregation of eight tetramers results in a filament subunit referred to as a unitlength filament (ULF) which has a length of about $60 \mathrm{~nm}$ (Herrmann et al., 1999). However, it is not yet fully understood which of the various oligomeric subunits contribute most substantially to the annealing process of filament formation in vivo (Herrmann et al., 2002; Herrmann and Aebi, 2004). We assume that the transition from the soluble pool to the filamentous compartment is initiated by dephosphorylation events starting with a pool of fully solubilized keratin oligomers. The resulting keratin filaments are represented by a track of connected line segments. Since each of the line segments models a filament building block consisting of 8 tetramers, the segment length is chosen as $45 \mathrm{~nm}$. This length is approximately gained when a $60 \mathrm{~nm}$ long ULF is added to a filament end, taking into account that longitudinal annealing involves an overlap of the ULFs participating. The intracellular distribution of soluble keratins is assumed to be governed by diffusion (Portet et al., 2003). The soluble pool is regarded as a concentration field on the observation window $W$. Keratin molecules transfer from the soluble into the filamentous pool during the course of network formation. Besides soluble pool consumption by network growth, the model assumes time evolution of the soluble pool to be controlled by a diffusion process, which 
is described by the following partial differential equation (PDE):

$$
\frac{\partial}{\partial t} C(t, x)=D\left(\frac{\partial^{2}}{\partial x_{1}^{2}} C(t, x)+\frac{\partial^{2}}{\partial x_{2}^{2}} C(t, x)\right)
$$

where $C(t, x)$ denotes the soluble pool concentration at time $t>0$ and at the location $x=$ $\left(x_{1}, x_{2}\right)$ in the interior int $W$ of the observation window; the diffusion constant $D>0$ determines the velocity of the diffusion process. Keratin molecules may leave as well as enter the observation window. Assuming a homogeneous soluble keratin concentration outside the observation window, this is reflected by imposing periodic boundary conditions for the diffusion PDE. These boundary conditions force keratin molecules which are leaving the observation window at a particular boundary location to reenter on the opposite side. Mathematically, by imposing periodic boundary conditions the solutions of the above PDE are required to satisfy

$$
C(t,(r, 0))=C(t,(r, l)) \text { and } C(t,(0, s))=C(t,(l, s)) \text { for all } r, s \in[0, l] \text {. }
$$

It can be shown that the above PDE with periodic boundary conditions has a unique solution for all of the bounded initial conditions $C(0, x)=f(x), x \in \operatorname{int} W$, arising in the context of our model. Moreover, the solution of the initial value problem is mass-preserving, i.e., the total amount of soluble keratin remains constant in time. Numerical solutions may be obtained by means of standard techniques such as finite difference schemes.

The initial state of the soluble pool is modeled as a constant tetramer concentration field on the observation window $W$. The total amount of soluble keratin at $t=0$ is given by $\int_{W} C(0, x) d x=$ $c l^{2}$.

For the configuration of the soluble and the filamentous pool at time $t \geq 0$ we introduce the notations $X_{t}^{(1)}$ and $X_{t}^{(2)}$, respectively. These random variables specify the state $X_{t}=\left(X_{t}^{(1)}, X_{t}^{(2)}\right)$ of the model at time $t \geq 0$. 


\subsection{Process of network formation}

\subsubsection{Mechanisms of network formation}

A first mechanism of network formation is filament nucleation, meaning the aggregation and annealing of small granule-like keratin particles, followed by an elongation process forming a new filament (Fig. 1A). Windoffer et al. (2004) report the observation of nucleation phenomena by means of fluorescence microscopy.

In our model, we assume the elongation of keratin filaments to be caused by longitudinal annealing of filament building blocks at the filament ends (Fig. 1B). Filament elongation has been studied in vitro by Herrmann and Aebi (2000) and Kirmse et al. (2007).

For the formation of the inter-filament connections, i.e. the nodes of the network, the model allows for simulating different mechanisms. Firstly, we consider the possibility of lateral annealing of soluble keratin along the existing filaments. Thus, it is assumed that network building blocks such as keratin tetramers are able to attach laterally along filamentous structures, thereby initiating a new network branch at the lateral annealing site (Fig. 1C). The corresponding vertex in the network graph is of degree 3, which means that three network segments emerge from this node, thus forming a Y-junction.

A second mechanism generating $Y$-junctions in the network is end-on integration of filament tips into the network, also referred to as merging (Fig. 1D). Merging has been observed in vivo by Windoffer et al. (2004). In electron microscopy images of keratin networks a certain fraction of the inter-filament connections are of degree 4. Therefore, once the growth trajectories of two filaments intersect, our model decides with a fixed merging probability $q$ whether merging occurs and a node of degree 3 is formed or a node of degree 4 is generated. In the sequel the latter event will be referred to as crossing (Fig. 1E). The parameter $q$ was chosen in a way that the relative frequencies of vertex degrees in the final network graphs were close to the values from our experimental data (Beil et al., 2005 and 2006).

\subsubsection{Time evolution of the network formation process}

The approach chosen to model the time evolution of the network is adapted to the high spatial resolution of the simulations. At high resolution, filament building blocks such as keratin tetramers 
and ULFs are rather large elongated objects. Network formation occurs whenever those elongated building blocks attach to the filament network. Times of network formation are described as a stochastic point process $\left\{\tau_{k}, k=1,2, \ldots\right\}$ on the positive real line. The random variables $\tau_{k}$ describe random times of network formation events, i.e., at these points in time filament building blocks transfer from the soluble pool into the filamentous pool by annealing. Keratin annealing is represented by the instantaneous addition of a new line segment to the segment system characterizing the network at the corresponding network formation time. Simultaneously, the soluble pool concentration is locally reduced by the amount consumed by network growth. Apart from these instantaneous local reductions of the soluble pool, the concentration fields of soluble keratin are regarded as permanently subjected to diffusion. Technically speaking, during the time intervals $\left(\tau_{k-1}, \tau_{k}\right), k=1,2, \ldots$, defining $\tau_{0}=0$, the diffusion PDE is applied to the initial concentration field $X_{\tau_{k-1}}^{(1)}$. For $k \geq 2$, the latter describes the soluble pool distribution right after the last network formation event, i.e., soluble pool consumption has already been incorporated. For $k \geq 1$, the distribution of the $\mathrm{k}$-th network formation time $\tau_{k}$ is determined by the distribution of the inter-occurrence times $\tau_{1}-\tau_{0}, \ldots, \tau_{k}-\tau_{k-1}$. For the random variable $\tau_{k}-\tau_{k-1}$ we assume a conditionally exponential distribution, given the state of the concentration field at time $\tau_{k-1}$ is $X_{\tau_{k-1}}^{(1)}=C$, i.e.,

$$
\mathbb{P}\left(\tau_{k}-\tau_{k-1} \leq t \mid X_{\tau_{k-1}}^{(1)}=C\right)=1-\exp (-t \lambda(C)) \text { for all } t>0
$$

This definition follows both, the Gillespie algorithm and the definition of a PDMP. By the properties of the exponential distribution, the state-dependent parameter $\lambda(C)>0$ describes the momentarily expected number of network formation events per unit time, given that the soluble pool is in state $C=\{C(x), x \in W\}$ (note that in contrast to $C(t, x)$ we only consider a particular spatial concentration field without time evolution). Since network formation is dominated by the elongation of a relatively small number of filament ends within the soluble keratin pool, we assume the reaction kinetics to be close to first order. Taking into account that 8 keratin tetramers are consumed for the formation of a single new filament segment, the following choice of $\lambda(C)$ ensures that the reaction follows a first order kinetics (Gillespie, 1977 and 1992):

$$
\lambda(C)=\frac{k}{8} \bar{C}
$$


where the reaction constant $k$ governs the velocity of the reaction and $\bar{C}=\int_{W} C(x) d x$ denotes the total amount of keratin in the concentration field $C$.

\subsubsection{Choice of the mechanism for network formation}

At any time $\tau_{k}$ of network formation, which has been determined by the stochastic point process $\left\{\tau_{k}\right\}$ introduced in Sec. 2.2.2, the mechanism of network formation needs to be specified. This is done in a two-step procedure that is illustrated in Fig. 2, part 1. First of all, based on certain conditional probabilities it is decided whether a new filament is initiated (event $A_{1}$ ) or an existing one elongates (event $A_{2}$ ). This step of the random decision process will be referred to as choice of the basic network growth type. Given that immediately before the event the soluble pool concentration field is $C$ and the set of filament ends of the current network configuration $\xi$ possibly elongating is $s(\xi)$, the following conditional probabilities are assigned to $A_{1}$ and $A_{2}$ :

$$
\begin{aligned}
& \mathbb{P}\left(A_{1} \mid X_{\tau_{k}-}=(C, \xi)\right)=\frac{M_{1} \bar{C}}{M_{1} \bar{C}+|s(\xi)|}, \\
& \mathbb{P}\left(A_{2} \mid X_{\tau_{k}-}=(C, \xi)\right)=\frac{|s(\xi)|}{M_{1} \bar{C}+|s(\xi)|},
\end{aligned}
$$

where $|s(\xi)|$ denotes the finite number of elements in $s(\xi)$. Note that by the notation $\tau_{k}-$ we refer to left limits of the state at the time $\tau_{k}$. The constant $M_{1}>0$ is a model parameter controlling the likelihood of those network formation events that are initiations of new filaments. By the above definition, the conditional probability of $A_{2}$, i.e., of a network formation event being filament elongation, is modeled to be increasing in the number $|s(\xi)|$ of filament ends possibly attracting filament building blocks for longitudinal annealing. Filament initiation (event $A_{1}$ ) includes nucleation as well as lateral annealing. Observations by Windoffer et al. (2004) suggest that the initiation of new filaments is preceded by the formation of small keratin clusters. Therefore, this event is assumed to require high levels of soluble keratin concentration. Consequently, the probability of $A_{1}$ is modeled to be decreasing with reduction of the amount $\bar{C}$ of soluble pool. In case the above random experiment has classified the basic growth type as filament initiation, a second random experiment determines whether nucleation (event $B_{1}$ ) or lateral annealing (event $B_{2}$ ) occurs. Lateral annealing is more likely to occur the more potential annealing sites are available. Therefore, the probability of lateral annealing is modeled to be increasing in the total length 
of the filament network. This is reflected by the following definition of the conditional probabilities, given the length $|\xi|$ of the network $\xi$ immediately before the network formation time $\tau_{k}$,

$$
\begin{aligned}
& \mathbb{P}\left(B_{1} \mid A_{1}, X_{\tau_{k}-}=(C, \xi)\right)=\frac{1}{1+M_{2}|\xi|}, \\
& \mathbb{P}\left(B_{2} \mid A_{1}, X_{\tau_{k}-}=(C, \xi)\right)=\frac{M_{2}|\xi|}{1+M_{2}|\xi|}
\end{aligned}
$$

where the constant $M_{2} \geq 0$ is a model parameter. Note that by means of the model parameters $M_{1}$ and $M_{2}$ the frequency of the single network formation mechanisms to occur can be controlled. In particular, it is possible to simulate scenarios without any lateral annealing by setting $M_{2}=0$.

\subsubsection{Choice of the location for network formation}

Once the mechanism of network formation has been determined, a location for keratin annealing, i.e. network building, needs to be specified (Fig. 2, part 2.). This is done according to a probability distribution of locations, which is based on the momentary distribution of soluble pool in the observation window. For this purpose, spatial probability fields are constructed such that potential network formation locations which are equipped with high local soluble pool concentrations are preferred sites of keratin annealing in comparison to those whose local concentrations are rather low. In the following let $b(z, \rho)$ denote the circle with radius $\rho$ centered at $z$. In case of an elongation (event $A_{2}$ ), and given the state of the system is $(C, \xi)$, a filament end $z \in s(\xi)$ is picked from the set $s(\xi)$ of all filament ends according to the following conditional probability:

$$
\mathbb{P}\left(z \mid A_{2}, X_{\tau_{k}-}=(C, \xi)\right)=\frac{\int_{b(z, \rho)} C(x) d x}{\sum_{y \in s(\xi)} \int_{b(y, \rho)} C(x) d x}
$$

In case of a nucleation (event $B_{1}$ ), the random location $z \in W$ is modeled to be distributed according to the conditional density

$$
f\left(z \mid B_{1}, X_{\tau_{k}-}=(C, \xi)\right)=\frac{\int_{b(z, \rho)} C(x) d x}{\int_{W} \int_{b(y, \rho)} C(x) d x d y} .
$$

The set of potential locations for lateral annealing (event $B_{2}$ ) is the filament network $\xi$ at the given network formation time. Thus, for determining a random site $z \in \xi$ for network formation 
given $B_{2}$, we introduce the following conditional density concentrated on the segment system $\xi$ representing the network:

$$
f\left(z \mid B_{2}, X_{\tau_{k}-}=(C, \xi)\right)=\frac{\int_{b(z, \rho)} C(x) d x}{\int_{\xi} \int_{b(y, \rho)} C(x) d x d y}
$$

The parameter $\rho$ defines the circular zone influencing the local probability of keratin annealing. Note that, following our periodic boundary approach, subsets of the circles $b(y, \rho)$ protruding the observation window are understood to be shifted to the opposite side of the window.

\subsubsection{Filament growth and soluble pool consumption}

Network growth is modeled as the instantaneous addition of small line segments to the existing network at the network formation times.

A quantitative investigation of keratin network morphology has been performed in electron microscopy images from the cortex of human cancer cells. The filaments in these compartments exhibited almost straight shapes (Beil et al., 2005). Bearing in mind the small persistence length of intermediate filaments, filament growth processes cannot be expected to directly account for this absence of curvature. However, to relate simulation results to real image data, we assumed straight filament elongation for our simulations, i.e., whenever a filament elongates there is no orientational deviation between the new line segment and the filament end it is appended to. Since electron microscopy data do not suggest the existence of preferred filament directions in keratin networks (Beil et al., 2005), the orientation of new line segments resulting from lateral annealing and nucleation is picked randomly according to the uniform distribution on $[0,2 \pi)$.

Corresponding to the periodic boundary conditions imposed on the soluble pool diffusion, for each filament leaving the observation window a new one is generated at the opposite side of the observation window. Orientations of the newly initiated filaments are assigned randomly. This boundary behavior of the filaments reflects the interaction of the observed part of the network with a homogenous environment.

End-on integration of a filament into the network (Fig. 1D) is modeled to occur with probability $q$, whenever the new segment intersects parts of the already existing network.

We will now specify the consumption of soluble keratin pool resulting from keratin annealing. Before soluble pool is consumed, a new line segment $\ell_{\tau_{k}}$ has been determined as described above. In 
the following, the dilation $\ell_{\tau_{k}} \oplus b(o, r)$ of $\ell_{\tau_{k}}$ denotes all points in the observation window whose distance to $\ell_{\tau_{k}}$ is no more than $r$. Parts of the segment dilations protruding the observation window are shifted to the opposite side.

Given that $C$ is the concentration field at a network reorganization time $\tau_{k}-$, there is a well defined dilation $\ell_{\tau_{k}} \oplus b(0, r)$ of the new line segment $\ell_{\tau_{k}}$ containing exactly the 8 tetramers that are assumed to form the new filament part. Technically speaking, given $C$ there is a dilation radius $r>0$ satisfying

$$
\int_{\ell_{\tau_{k}} \oplus b(0, r)} C(x) d x=8,
$$

provided that $\bar{C} \geq 8$. Note however, that in our simulations the pathological case $\bar{C}<8$, i.e., of concentration fields not containing enough tetramers to find these dilation radii, does not occur and was therefore neglected. We define the concentration field immediately after network formation by

$$
X_{\tau_{k}}^{(1)}(x)=\left\{\begin{array}{cl}
0 & \text { if } x \in \ell_{\tau_{k}} \oplus b(0, r) \\
X_{\tau_{k}-}^{(1)}(x) & \text { else. }
\end{array}\right.
$$

Note that this concentration field serves as the initial condition for soluble pool diffusion after time $\tau_{k}$.

Since protein synthesis is neglected, and phosphorylation events are not modeled, network formation is considered to be complete when the soluble pool concentration has fallen below a critical level that does not allow for any more substantial polymerization.

For investigations of network architecture concerning the mean number of network vertices and edges it must be taken into account that keratin filaments have a certain width, whereas their model representation as line segments reduces them to their longitudinal axis. For this reason, vertices located closely to each other $(<20 \mathrm{~nm})$ should not be interpreted as distinct, i.e. they have to be contracted. A recursive procedure is applied for this task, which has been established in Beil et al. (2005).

\section{Simulations and analysis of network architecture}

The aim of this study was to investigate the effects of distinct network formation mechanisms as controlled by model parameters (Tab. 1) on the morphology of keratin networks. For this purpose 
a simulation algorithm was implemented in Java within the GeoStoch software library (Mayer et al., 2004).

\subsection{Settings}

The observation window was discretized by a grid of $500 \times 500$ pixels with a spatial resolution of $5.3 \mathrm{~nm}$ per pixel length. Given this grid, the solution of the diffusion PDE was numerically approximated by means of a finite difference scheme.

The simulation outputs, i.e. the final states of the network, were given as binary images.

Due to the transformation of the tetramer concentration fields representing the soluble pool into spatial probability fields at each time of network formation, simulations were computationally demanding. Simulation times totaled around $1.5 \mathrm{~h}$ per run on an AMD Opteron 252 processor (2.6GHz, $8 G B$ RAM).

For each parameter constellation considered, we conducted 30 simulation runs in order to control for stochastic variability between different runs.

Standard settings were chosen as depicted in Tab. 2. Based on this standard scenario model parameters were varied in order to investigate their effects on network morphology.

The reaction constant $k$ was chosen such that the whole network formation process was finished after around 15 minutes, which is a time span found to separate the time of maximal keratin phosphorylation and reestablishment of the network when keratin networks are exposed to a phosphorylation pulse (Beil et al., 2005). Network formation was considered to be finished as soon as the mean soluble pool concentration had fallen below 150 tetramers per $\mu m^{2}$.

\subsection{Model parameters to be varied}

Apart from the concentration $c$ of the initial soluble pool, which determines the amount of building material for the network and was estimated from the mean network length per unit area in images of cancer cells (Beil et al., 2006), the key parameters $M_{1}$ and $M_{2}$, which control the likelihood of specific network formation mechanisms, were varied. Note that $M_{1}$ affects the choice of the basic network growth type, i.e. the probability of a network formation event being a filament initiation rather than the elongation of an existing filament. In case of a filament initiation, the parameter $M_{2}$ controls the probability of this initiation being a lateral annealing event rather than a free 
nucleation. Whenever one of the above parameters was varied, the others remained fixed, their values being set to $c=6200, M_{1}=0.002$ and $M_{2}=0.22$.

\subsection{Statistical analysis of network characteristics}

First order characteristics such as the mean number of network nodes (i.e. vertices whose degree was at least 3), the mean number of edges and the mean number of meshes were considered. Each of these quantities was estimated with respect to the unit area. We also considered features of network connectivity and second order characteristics to evaluate clustering tendencies of the network meshes.

\subsubsection{Connectivity}

One of the objectives of our statistical analysis was the assessment of the efficiency of a network formation scenario in establishing inter-filament connectivity, which is a pertinent feature of networks that determines their mechanics (Blumenfeld, 2006; Huisman et al., 2007). To obtain a measure for connectivity, a Euclidian minimum spanning tree (MST) was computed for each connectivity component of the network graph using Prim's algorithm (Jungnickel, 1999). An MST of a fully connected graph is a subgraph with the same set of vertices where the latter are connected by line segments from the original graph's edge set in such a way that the total length of all the edges is minimal while any vertex can still be reached from any other by following the edges. After the minimum spanning trees had been constructed for each connectivity component, their lengths were added and divided by the length of the entire graph. This quotient will be referred to as the relative MST-length. It is a measure for redundancy in the network graph. Highly connected networks exhibit a low relative MST-length since a high percentage of their edges can be discarded without destroying the connectivity of the graph. On the other hand, in networks with low connectivity many of the edges could only be removed at the cost of disconnecting the network. Therefore a high percentage of the original edges is still contained in the MST and hence the relative MST-length is relatively large. 


\subsubsection{Pair-correlation function of mesh centers}

Network clustering was studied by considering second order statistics of network meshes. First, a center point was attributed to each mesh by taking the center of a circle with maximum possible radius to be inscribed into this mesh. The in-circles were determined after truncation of all dead ends in the network graph, since the latter do not contribute to the constitution of the meshes and are mechanically not relevant. Mesh clustering was quantified by the analysis of clustering effects in the point patterns of mesh centers. For this purpose we determined the empirical pair correlation function $\widehat{g}(r), r>0$ of the mesh centers (Stoyan and Stoyan, 1994). Note that the theoretical pair-correlation function of a homogeneous Poisson-type point pattern, which is a model for complete spatial randomness (without clustering), is constant and equals 1 . Values of $\widehat{g}(r)$ greater than 1 indicate that point pairs of distance $r$ occur rather frequently, whereas values of $\widehat{g}(r)$ smaller than 1 occur if point pairs with this distance are relatively rare. Networks exhibiting clusters of small meshes will thus show a peak of their empirical pair correlation function at small distances $r$. Given a point pattern $\left\{S_{1}, \ldots, S_{m}\right\}$, the definition of $\widehat{g}(r)$ is as follows:

$$
\widehat{g}(r)=\frac{1}{(m /|W|)^{2}} \sum_{n_{1} \neq n_{2}} \frac{K\left(\left\|S_{n_{2}}-S_{n_{1}}\right\|-r\right) \mathbb{I}_{W \times W}\left(S_{n_{1}}, S_{n_{2}}\right)}{2 \pi r\left|W \cap\left(W+\left(S_{n_{2}}-S_{n_{1}}\right)\right)\right|}
$$

where $\|x-y\|$ is the distance between mesh centers $x$ and $y, W+x$ denotes the observation window shifted by the vector $x$ and $|B|$ the area of a set $B \subset \mathbb{R}^{2} . K$ denotes the Epanechnikov kernel (Stoyan and Stoyan, 1994). The bandwidth for $K$ was chosen as $0.15 / \sqrt{\widehat{\lambda}}$, where $\hat{\lambda}$ is the mean number of mesh centers per unit area. The empirical pair-correlation functions for the simulated data were obtained by distance-wise averaging of the estimators over all 30 simulation runs that were performed for each of the scenarios.

\section{Simulation results}

For each parameter constellation, the results of the 30 simulation runs performed were visualized by boxplots. Regression lines and curves were fitted to the mean values.

All sample images displayed were randomly selected among the simulation runs. 


\subsection{Role of the initial soluble pool concentration $c$ for network architecture}

Fig. 3, 4A, and 4B show the response of the network morphology if the concentration $c$ of soluble keratin at the beginning of the simulation was varied in steps of 500 tetramers per $\mu m^{2}$ over the interval $[1500,7500]$.

Note that the mean number of edges, meshes and network nodes increased with $c$. Network connectivity increased with the background concentration since the relative MST-length decayed exponentially (Fig. 4B). As a consequence, the efficiency in establishing network connectivity was almost unaffected by variations of $c$ as soon as a critical level of 3500 tetramers per $\mu m^{2}$ was exceeded.

The pair-correlation function did not indicate a major effect of $c$ on the clustering tendency of network meshes (Fig. 9A). Note that for $c<3500$ the point pattern of mesh centers did not contain enough points for a reliable estimation of the pair-correlation function.

\subsection{Role of filament initiation type for network architecture}

In our model, initiation of new filaments during network formation could either occur as a nucleation in free space or as lateral keratin annealing at an existing filament (Fig. $1 \mathrm{~A}$ and $1 \mathrm{C}$ ). The probability of the latter was controlled by the model parameter $M_{2}$. Sample images of simulations for different values of $M_{2}$ can be seen in Fig. 5.

Fig. 4C shows that increasing the probability of lateral annealing hardly changed the mean-value characteristics of the network. Furthermore, the efficiency in establishing network connectivity was also almost unaffected by variations of $M_{2}$ (Fig. 4D).

The main effect of lateral annealing was an increased tendency of the network to form microgel structures, i.e., clusters of small network meshes. If $M_{2}$ was increased, the empirical pair correlation function $\widehat{g}(r)$ indicated a substantial rise in likelihood of mesh center distances between 50 and $150 \mathrm{~nm}$ (Fig. 9B). Even without any lateral annealing, i.e., in case $M_{2}=0$, the peak of $\widehat{g}(r)$ indicated a small clustering effect of the meshes. Once $M_{2} \geq 1.0$, the effect of further increases of $M_{2}$ on mesh clustering subsided (besides Fig. 9B see also the simulation results in Fig. 5) . 


\subsection{Role of basic growth type for network architecture}

By construction of our model, at each network formation time the outcome of a Bernoulli experiment determined the basic type of network growth, i.e., wether a new filament was initiated or an existing one elongated. Increasing the parameter $M_{1}$ resulted in a higher probability for filament initiation in relation to the elongation probability of the existing filaments. Sample simulation results are displayed in Fig. 6. The mean numbers of edges and network vertices were hardly affected by variations of $M_{1}$ (Fig. 4E).

However, network connectivity was harmed if by an increase of $M_{1}$ the elongation tendency of the filaments was diminished. This was indicated by the linear growth behavior of the relative MST-length (Fig. 4F). Furthermore, the mean number of network meshes substantially decreased with growing $M_{1}$ (Fig. 4E). Thus, the ability of the filaments to form meshes was reduced if the elongation tendency of the filaments was diminished in favor of more filament initiations. Considering Fig. 7, which displays simulated networks for large values of $M_{1}$, namely $M_{1}=0.016$ and $M_{1}=0.032$, the negative impact of decreased filament elongation tendencies on network connectivity becomes ostensive, since in these cases the keratin tended to form filament clusters rather than a homogeneous network. Notice that both the mean number of network meshes and the relative MST-length appeared to be hardly affected if small values of $M_{1}$ were considered, i.e. $M_{1} \in[0.004,0.0055]$. A substantial linear growth behavior occurred only for $M_{1} \geq 0.006$.

Besides its effect on network connectivity, $M_{1}$ also affected mesh clustering. If $M_{1}$ was increased, the empirical pair correlation function $\widehat{g}(r)$ indicated a substantial rise in likelihood of mesh center distances between 50 and $150 \mathrm{~nm}$ (Fig. 9C).

\subsection{Interaction of parameters $M_{1}$ and $M_{2}$}

As soon as a network formation event had been classified as filament initiation, it was decided according to a state-dependent discrete distribution if the new filament was initiated by lateral annealing or by free nucleation in the cytoplasm. Given a filament initiation event, the conditional probability for lateral annealing was controlled by the parameter $M_{2}$. As a consequence of this modeling approach, not only an increase of the parameter $M_{2}$ but also an increase of $M_{1}$ resulted in a higher mean number of lateral annealing events as soon as $M_{2}>0$. Therefore, simulations were performed in order to clarify the impact of lateral annealing on mesh clustering as well as 
network connectivity. More precisely, we compared the above simulations for variations of $M_{1}$, which included lateral annealing since $M_{2}=0.22>0$, to the case where lateral annealing was excluded by setting $M_{2}=0$. Sample simulation outputs can be found in Fig. 8 .

The response of network connectivity to variations of $M_{1}$ occurred independently of lateral annealing; if lateral annealing was excluded by setting $M_{2}=0$, the relative MST-length behaved similarly to the standard scenario.

The impact of $M_{1}$ on mesh clustering was however sensitive to the choice of $M_{2}$. Fig. 9D shows that in case $M_{2}=0$, clustering effects were not only less pronounced but unaffected by variations of $M_{1}$, i.e., of the nucleation intensity (see also Fig. 8). Thus, in our simulations the network formation mechanism of lateral annealing solely accounted for pronounced mesh clustering, i.e. the formation of microgel structures.

\subsection{Role of diffusion}

Visualizations of concentration fields of soluble oligomers as presented in Fig. 10 illustrate that low diffusion coefficients can lead to a localized depletion of the soluble pool. For these simulations parameter values were selected in a way, that promoted mesh clustering and a high network connectivity $\left(M_{1}=0.012, M_{2}=0.22\right)$. Both scenarios are associated with a fast soluble pool consumption at a local level and, thus, were expected to result in a maximum depletion of soluble oligomers. However, depletion zones and thus diffusion limitations disappeared for a diffusion coefficient $D \geq 8 \times 10^{-4} \mu \mathrm{ms}^{-1}$, which is still a magnitude smaller than theoretical values calculated by Portet et al. (2003). Thus, under these conditions the system can be regarded as reaction-limited.

The density of nodes, meshes and edges did not change if $D$ was varied between $5 \times 10^{-5}$ and $3.2 \times 10^{-3} \mu \mathrm{ms}^{-1}$. In contrast, clustering was influenced by very small values for $D$, which, however, are more than a magnitude smaller than the values estimated by Portet et al. (2003) (Fig. 11). 


\section{Discussion}

Whereas many details of the filament assembly and network formation processes for the actin and microtubular cytoskeleton are now well understood (Raynaud-Messina and Merdes, 2007; Schaus et al., 2007) most of the mechanisms involved in the synthesis of keratin intermediate filament networks still remain elusive. Thus, quantitative models can be useful to identify and characterize mechanisms which determine the architecture of these networks. The comparison of keratin networks simulated in this study with such from electron microscopy images of cancer cells emphasizes this fact (Figure 10). Models developed to investigate the formation of networks of actin filaments or microtubules are based on the specific biochemical properties of these filament systems. Due to the polarity of filament growth and restrictions for the angle of filament branching, such models are anisotropic in nature (Maly and Borisy, 2001; Haviv et al., 2006). A recent paper by Fass et al. (2008) presented a Gillespie-like model to investigate actin filament fragmentation and annealing, but did not analyze these issues in the context of network architecture. Although actin filament dynamics are well studied it still appears to be difficult to address the issue of actin network architecture at all necessary scales (Mogilner, 2006). Nevertheless, several aspects of our model can be regarded as simplifications of existing models for actin networks. Apart from the lack of filament polarity, there is no restriction for the angles of filament branching or interactions as observed by Windoffer et al. (2004) in living cells. Consequently, keratin networks can be isotropic and fill the cytoplasm without the need to align with the other (polarized) networks. Intermediate filament networks can thereby fulfill their fundamental role as integrators of cells in a mostly independent way (Lazarides, 1980). Moreover, since new building blocks can be added at any place along existing filaments, mesh clusters can easily be produced. Thus, keratin networks could represent a fast and energy-efficient buffer system for mechanical stress regardless of its direction and location.

The new approach to model the spatiotemporal distribution of the molecular events of keratin network assembly reflects both, the incomplete understanding of the biological mechanisms involved and the probabilistic nature of a spatiotemporal reaction system at different scales, i.e. the scale of soluble keratin oligomers and of filaments. Although the number of soluble keratin oligomers is large enough to use a PDE to model diffusion, this process is also stochastic and could be modeled as a set of random walks. However, the latter approach is computationally not feasible for higher 
concentrations. The timing of network growth events, which are infrequent compared to oligomer movements, was modeled by a time-continuous Markov process. This method is well established to model chemical reactions (Gillespie 1977) and favorable in situations where the number of molecules involved is rather small for some of the reacting species and deterministic approaches become questionable (Gillespie, 1977 and 1992; Cao et al., 2005). Gillespie's algorithm is based on the assumption that the distribution of the inter-occurrence times between single reactions only depends on the state of the system immediately after the last reaction event. The design of a piecewise-deterministic Markov process leaves this essential property unchanged. This approach makes these models particularly efficient for computer simulation and is an essential advantage in comparison to other stochastic processes such as general Lévy processes or stochastic differential equations whose simulation is usually based on interpolation of discrete skeletons and thus requires analysis of approximation errors (Asmussen and Glynn, 2007).

For investigating the formation of keratin networks, the spatial structure of the reaction system was at least as important as global reaction kinetics, the latter being modeled as being first order, since the dominant component of the reaction was given by the elongating filament ends. Consequently, the state space of our stochastic process was chosen a hybrid of a geometric space, namely the family of line segment systems, and a function space, modeling concentration fields of soluble keratin oligomers in the observation window. Thus, the model differs from classical Gillespie-type processes, whose states simply describe the numbers of molecules for all species in the systems at a given time instead of monitoring the spatial configuration of the system. Moreover, whereas the Gillespie algorithm leaves the state of the system unchanged between reaction events, our model also captures the spatial evolution of the soluble pool between network formation events. The Gillespie-type component was solely used to determine the times of network formation. Locations of network formation were assigned based on the spatial distribution of the soluble keratin pool fueling network growth. The time evolution of the soluble pool between network growth events, which consume soluble oligomers, was modeled by a deterministic diffusion equation. This model component constitutes a mean-value approach to what would have been obtained by modeling Brownian-type movements of single molecules in the soluble pool. For the choice of network formation locations, the concentration fields were interpreted as spatial probability fields. This approach takes into account uncertainty about the spatial distribution of the soluble keratin pool and reflects the stochastic nature of the underlying physical process. Investigation of the concen- 
tration fields during simulations allows for an easy assessment of diffusion limitations arising for network formation scenarios of interest. This way, the model provides the opportunity to study the interplay between diffusion, network growth events and the resulting network morphology, but requires a more complex structure than simple Monte Carlo methods. Other situations where stochastic models for chemical reaction systems have been combined with deterministic components are discussed in Haseltine and Rawlings (2002).

Since the information about keratin network formation is scarce we restricted the set of possible network formation mechanisms in our model to processes that can be verified in experiments. It includes filament nucleation and elongation, lateral annealing and merging of filaments. Some of these processes were already observed in-vitro or in-vivo (Windoffer et al., 2004; Kirmse et al., 2007). These processes represent discrete events in space and time at the scales regarded in our simulations. The mathematical model for the built up of the network was designed in a way that permitted one event per time point, the latter being determined by a point process on the positive real line. The selection of network formation mechanisms for the events was controlled by only two parameters of the model, namely $M_{1}$ and $M_{2}$. In the simulation studies, we investigated the impact of these two parameters and the initial background concentration $c$ on network morphology. Simulation outputs suggest that the background concentration may be viewed as a scaling parameter of network mean value characteristics, which grew almost linearly. The relative MST-length decayed exponentially, thus, well connected networks were established once a concentration of 3500 tetramers $/ \mu m^{2}$ was exceeded. Network connectivity was not influenced by the parameter $M_{2}$ controlling the frequency of lateral annealing events (Fig. 4D), whereas it seems to be substantially dependent on the relation between filament elongation and initiation events, which was determined by $M_{1}$ (Fig. 4F). High network connectivity seems to be favored by rapid elongation of the filaments once they have been initiated. Rapid filament elongation has also been observed in vivo by Windoffer et al. (2004) and may thus be a key factor for the high degree of connectivity found in image data of keratin networks. This process, however, might be restricted by the diffusion-limited supply with soluble keratin oligomers. However, the diffusion coefficient for soluble oligomers has to be very small to create a system which is diffusion-limited. Even in the absence of experimental data for intracellular keratin diffusion coefficients, theoretical values estimated by Portet et al.(2003) are much greater than the threshold for the switch from a reaction- to a diffusion-limited system. Thus, it is fair to assume that keratin network synthesis is 
reaction-limited. However, experimental studies investigating the intracellular diffusion of keratin oligomers are required to verify this hypothesis.

Our simulation experiments revealed lateral annealing to be a mechanism that controls the formation of mesh clusters. Clustering tendencies of network meshes can also be observed in electron microscopy data of keratin networks (Beil et al., 2005). In order to explain the impact of lateral annealing on clustering effects, series of images displaying the network at different times throughout its formation process were generated. These show that enhancing the likelihood of lateral annealing in comparison to free nucleation favored the formation of several spatially segregated centers of keratin annealing activity. This was due to the fact that the frequently occurring lateral annealing events were confined to those locations already occupied by filaments. By the end of network formation, these annealing centers exhibited a microgel structure. On the other hand, if the likelihood of lateral annealing was reduced in favor of free nucleation, new filaments were initiated evenly spread out over the observation window. As a consequence, neither preferred sites of network formation nor pronounced microgel structures emerged.

In the standard setting of parameters the pair-correlation function also indicated pronounced mesh clustering when the parameter $M_{1}$ controlling the frequency of filament initiations was increased. This is plausible since for each filament initiation the model decided randomly if the new filament was initiated by lateral annealing or by free nucleation in the cytoplasm. Since the standard setting of parameters allowed for lateral annealing $\left(M_{2}=0.22\right)$, an increase of $M_{1}$ resulted in a higher mean number of lateral annealing events. Having identified lateral annealing as a mechanism controlling mesh clustering, the similar effect of the parameters $M_{1}$ and $M_{2}$ on the pair-correlation function (Fig. 9C and 9B) was predictable. On the other hand, simulations without lateral annealing $\left(M_{2}=0\right)$ revealed that free nucleation events did not affect the pair-correlation function (Fig. 9D). Thus, in the simulations mesh clustering was purely a contribution of lateral annealing. The architecture of the keratin cytoskeleton plays a pivotal role for cell migration by regulating cell viscoelasticity (Beil et al., 2003). The classical models for determining mechanical properties of biopolymer networks relate the mean mesh size to the elastic shear modulus (MacKintosh et al., 1995). Structural homogeneity and isotropy constitutes prerequisites for this approach (Storm et al., 2005). Since these conditions do not appear to be applicable to intracellular keratin filament networks, our model is not restricted to produce this type of networks. Consequently, we did not focus our descriptive analysis on mean mesh sizes but on complex structural features such 
as connectivity and clustering processes. By modulating connectivity cells may control the transduction of forces and information (Blumenfeld, 2006). Clustering processes within the non-polar network, as regulated by branching, provide cells with the opportunity to adapt to local demands without changing the total amount of keratin proteins. Finite element modeling may be applied to establish a relationship between these features and network mechanics by determining global as well as local mechanical properties of simulated networks (Heussinger and Frey, 2006). This way, molecular events which govern the biophysical features of the cytoskeleton can be identified in simulation experiments.

\section{Acknowledgment}

This work was supported by grants from Deutsche Forschungsgemeinschaft to MB and VS (SFB 518 projects B21 and B22). SP was supported by NSERC Discovery Grant. We thank Stefan Funken for his help in numerically solving the diffusion PDE. 


\section{References}

[1] Asmussen, S., Glynn, P.W. 2007. Stochastic simulation. Algorithms and analysis. Springer, New York.

[2] Ballestrem, C., Wehrle-Haller, B., Hinz, B., Imhof, B.A. 2000. Actin-dependent lamellipodia formation and microtubule-dependent tail retraction control directed cell migration. Mol. Biol. Cell 11, 2999-3012.

[3] Beil, M., Micoulet, A., von Wichert, G., Paschke, S., Walther, P., Omary, M.B., Van Veldhoven, P.P., Gern, U., Wolff-Hieber, E., Eggermann, J., Waltenberger, J., Adler, G., Spatz, J., Seufferlein, T. 2003. Sphingosylphosphorycholine regulates keratin network architecture and visco-elastic properties of human cancer cells. Nat. Cell Biol. 5, 803-811.

[4] Beil, M., Braxmeier, H., Fleischer, F., Schmidt, V., Walther, P. 2005. Quantitative analysis of keratin filament networks in scanning electron microsopy images of cancer cells. J. Microsc. 220, 84-95.

[5] Beil, M., Eckel, S., Fleischer, F., Schmidt, H., Schmidt, V., Walther, P. 2006. Fitting of random tessellation models to keratin filament networks. J. Theor. Biol. 241(1), 62-72.

[6] Blumenfeld, R. 2006. Isostaticity and controlled force transmission in the cytoskeleton: a model awaiting experimental evidence. Biophys. J. 91, 1970-1983.

[7] Cao, Y., Gillespie, D.T., Petzold, L.R. 2005. The slow-scale stochastic simulation algorithm. J. Chem. Phys. 122, 014116.

[8] Coulombe, P.A., Omary, M.B. 2002. 'Hard' and 'soft' principles defining the structure, function and regulation of keratin intermediate filaments. Curr. Opin. Cell Biol. 14(1), 110-122.

[9] Coulombe, P.A., Wong, P. 2004. Cytoplasmic intermediate filaments revealed as dynamic and multipurpose scaffolds. Nat. Cell Biol. 6(8), 699-706.

[10] Davis, M.H.A. 1984. Piecewise-deterministic Markov processes: a general class of nondiffusion stochastic models. J. Roy. Stat. Soc. B 46(3), 353-388.

[11] Fass, J., Pak, C., Bamburg, J., Mogilner, A. 2008. Stochastic simulation of actin dynamics reveals the role of annealing and fragmentation. J. Theor. Biol. 252(1), 173-183. 
[12] Fleischer, F., Ananthakrishnan, R., Eckel, S., Schmidt, H., Käs, J., Svitkina, T., Schmidt, V., Beil, M. 2007. Actin network architecture and elasticity in lamellipodia of melanoma cells. New J. Phys. 9, 420.

[13] Geisler, N., Schunemann, J., Weber, K., Haner, M., Aebi, U. 1998. Assembly and architecture of invertebrate cytoplasmic intermediate filaments reconcile features of vertebrate cytoplasmic and nuclear lamine-type intermediate filaments. J. Mol. Biol. 282, 601-617.

[14] Gillespie, D.T. 1977. Exact stochastic simulation of coupled chemical reactions. J. Phys. Chem. 81(25), 2340-2361.

[15] Gillespie, D.T. 1992. A rigorous derivation of the chemical master equation. Physica A 188, 404-425.

[16] Haseltine, E.L., Rawlings, J.B. 2002. Approximate simulation of coupled fast and slow reactions for stochastic chemical kinetics. J. Chem. Phys. 117(15), 6959-6969.

[17] Haviv, L., Brill-Karniely, Y., Mahaffy, R., Backouche, F., Ben-Shaul, A., Pollard, T.D., Bernheim-Groswasser, A. 2006. Reconstitution of the transition from lamellipodium to filopodium in a membrane-free system. Proc. Natl. Acad. Sci. USA 103(13), 4906-4911.

[18] Herrmann, H., Haner, M., Brettel, M., Ku, N.O., Aebi, U. 1999. Characterization of distinct early assembly units of different intermediate filament proteins. J. Mol. Biol. 286(5), 14031420.

[19] Herrmann, H., Aebi, U. 2000. Intermediate filaments and their associates: multi-talented structural elements specifying cytoarchitecture and cytodynamics. Curr. Opin. Cell Biol. 12, 79-90.

[20] Herrmann, H., Wedig, T., Porter, R.M., Lane, E.B., Aebi, U. 2002. Characterization of early assembly intermediates of recombinant human keratins. J. Struct. Biol. 137(1-2), 82-96.

[21] Herrmann, H., Aebi, U. 2004. Intermediate filaments: molecular structure, assembly mechanism, and integration into functionally distinct intracellular scaffolds. Annu. Rev. Biochem. 73, 749-789.

[22] Herrmann, H., Bär, H., Kreplak, L., Strelkov, S.V., Aebi, U. 2007. Intermediate filaments: from cell architecture to nanomechanics. Nat. Rev. Mol. Cell Biol. 8(7), 562-573. 
[23] Heussinger, C., Frey, E. 2006. Stiff polymers, foams, and fiber networks. Phys. Rev. Lett. 96, 017802.

[24] Huisman, E.M., van Dillen, T., Onck, P.R., van der Giessen, E. 2007. Three-dimensional cross-linked F-actin networks: relation between network architecture and mechanical behavior. Phys. Rev. Let. 99, 208103.

[25] Jungnickel, D. 1999. Graphs, networks and algorithms. Springer, Berlin.

[26] Kirmse, R., Portet, S., Mucke, N., Aebi, U., Herrmann, H., Langowski, J. 2007. A quantitative kinetic model for the in vitro assembly of intermediate filaments from tetrameric vimentin. J. Biol. Chem. 282, 18563-18572.

[27] Lazarides, E. 1980. Intermediate filaments as mechanical integrators of cellular space. Nature 283, 249-255.

[28] MacKintosh, F., Käs, J., Janmey, P. 1995. Elasticity of semiflexible polymer networks. Phys. Rev. Lett. 75, 4425-4428.

[29] Magin, T.M., Vijayaraj, P., Leube, R.E. 2007. Abstract structural and regulatory functions of keratins. Exp. Cell Res. 313(10), 2021-2032.

[30] Maly, I.V., Borisy, G.G. 2001. Self-organization of a propulsive actin network as an evolutionary process. Proc. Natl. Acad. Sci. USA 98(20), 11324-11329.

[31] Mayer, J., Schmidt, V., Schweiggert, F. 2004. A unified simulation framework for spatial stochastic models. Simul. Model. Pract. Th. 12, 307-326.

[32] Mogilner, A., Wollman, R., Marshall, W.F. 2006. Quantitative modeling in cell biology: What is it good for?. Dev. Cell. 11, 279-287.

[33] Moll, R., Divo, M., Langbein, L. 2008. The human keratins: biology and pathology. Histochem. Cell Biol. 129(6), 705-733.

[34] Morse, D.C. 1998. Viscoelasticity of concentrated isotropic solutions of semiflexible polymers: 1. Model and stress tensors. Macromolecules 31, 7030-7043.

[35] Omary, M.B., Ku, N., Tao, G., Toivola, D.M., Liao, J. 2006. 'Heads and tails' of intermediate filament phosphorylation: multiple sites and functional insights. Trends Biochem. Sci. 31(7), 383-394. 
[36] Oshima, R.G. 2007. Intermediate filaments: a historical perspective. Exp. Cell Res. 313, 1981-1994.

[37] Portet, S., Arino, O., Vassy, J., Schoevaert, D. 2003. Organization of the cytokeratin network in an epithelial cell. J. Theor. Biol. 223, 313-333.

[38] Raynaud-Messina, B., Merdes, A. 2007. y-tubulin complexes and microtubule organization. Curr. Opin. Cell Biol. 19(1), 24-30.

[39] Schaus, T.E., Taylor, E.W., Borisy, G.G. 2007. Self-organization of actin filament orientation in the dendritic-nucleation/array-treadmilling model. Proc. Natl. Acad. Sci. USA 104(17), 7086-7091.

[40] Storm, C., Pastore, J.J., MacKintosh, F.C., Lubensky, T.C., Janmey, P.A. 2005. Nonlinear elasticity in biological gels. Nature 435, 191-194.

[41] Stoyan, D., Stoyan, H. 1994. Fractals, random shapes and point fields. Methods of geometrical statistics. John Wiley and Sons, Chichester.

[42] Strnad, P., Windoffer, R., Leube, R.E. 2002. Induction of rapid and reversible cytokeratin network remodeling by inhibition of tyrosine phosphatases. J. Cell Sci. 115, 4133-4148.

[43] Wagner, O.I., Rammensee, S., Korde, N., Wen, Q., Leterrier, J.F., Janmey, P.A. 2007. Abstract softness, strength and self-repair in intermediate filament networks. Exp. Cell Res. 313(10), 2228-2235.

[44] Windoffer, R., Woll, S., Strnad, P., Leube, R.E. 2004. Identification of novel principles of keratin filament network turnover in living cells. Mol. Biol. Cell 15, 2436-2448. 


\begin{tabular}{|l|l|}
\hline$l$ & length of the observation window \\
\hline$D$ & diffusion constant \\
\hline$k$ & reaction constant for keratin annealing \\
\hline$\rho$ & $\begin{array}{l}\text { radius of the circle whose soluble pool determines } \\
\text { the probability of local keratin annealing }\end{array}$ \\
\hline$q$ & probability for end-on integration \\
\hline $\mathrm{C}$ & $\begin{array}{l}\text { background concentration in keratin tetramers per } \\
\mu m^{2}\end{array}$ \\
\hline$M_{1}$ & $\begin{array}{l}\text { controls the probability of filament initiation in } \\
\text { comparison to filament elongation }\end{array}$ \\
\hline$M_{2}$ & $\begin{array}{l}\text { controls the probability of lateral annealing in com- } \\
\text { parison to free filament nucleation }\end{array}$ \\
\hline
\end{tabular}

Table 1: Model parameters

\begin{tabular}{|l|l|l|}
\hline$l$ & length of the observation window & $2.65 \mu \mathrm{m}(500$ pixels $)$ \\
\hline$D$ & diffusion constant & $0.0005 \mu \mathrm{m}^{2} \mathrm{~s}^{-1}$ \\
\hline$k$ & reaction constant for keratin annealing & $0.004 /($ tetramer $\cdot s)$ \\
\hline$\rho$ & $\begin{array}{l}\text { radius of the circle whose soluble pool determines } \\
\text { the probability of local keratin annealing }\end{array}$ & $25 \mathrm{~nm}$ \\
\hline$q$ & probability for end-on integration & 0.8 \\
\hline
\end{tabular}

Table 2: Standard settings for the simulations 

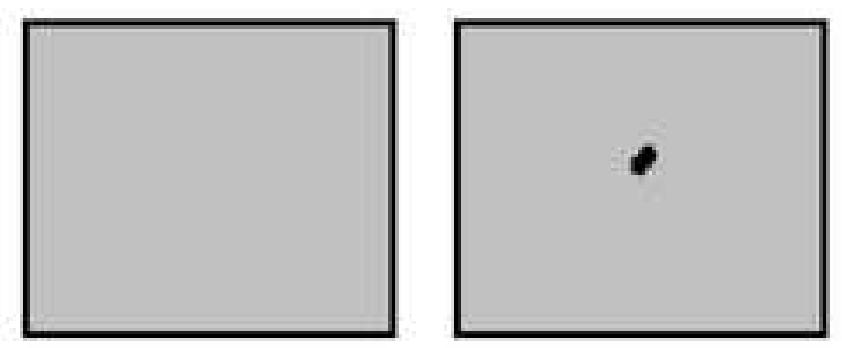

A Nucleation of a filament
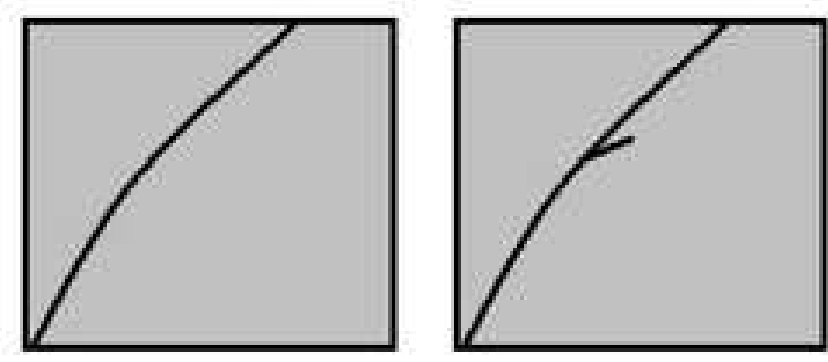

C Lateral keratin annealing
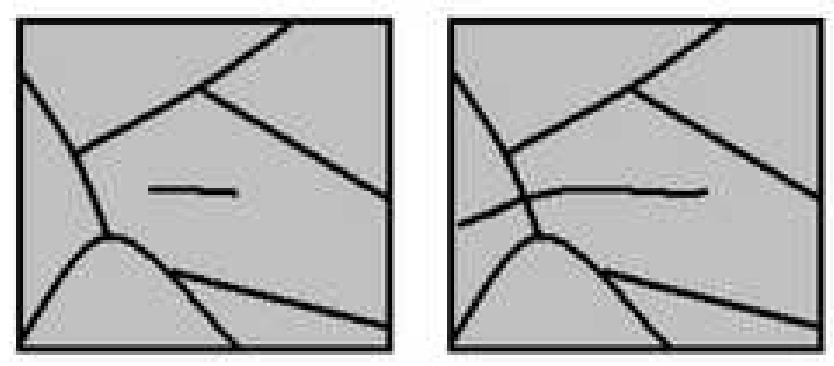

E Crossing of two filaments

Figure 1: Mechanisms of network formation

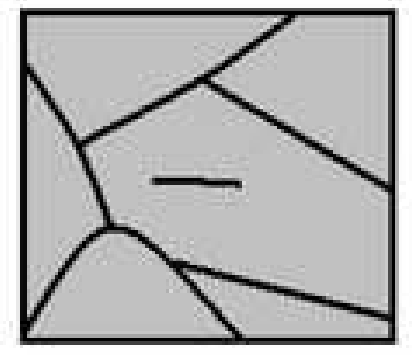
work

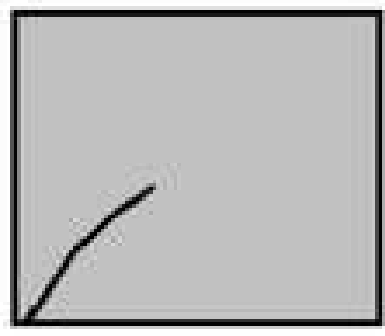

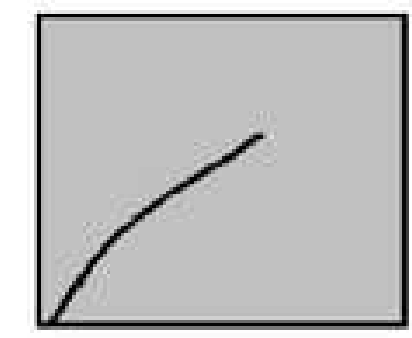

B Elongation of a filament

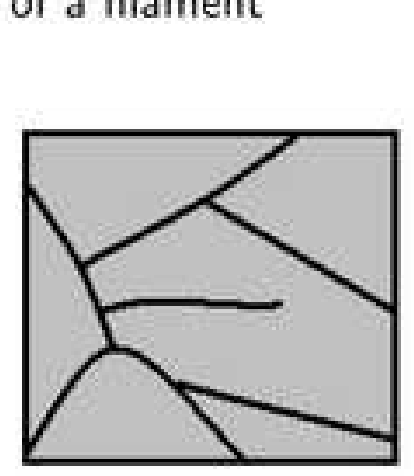

D Merging of a filament end with the net- 


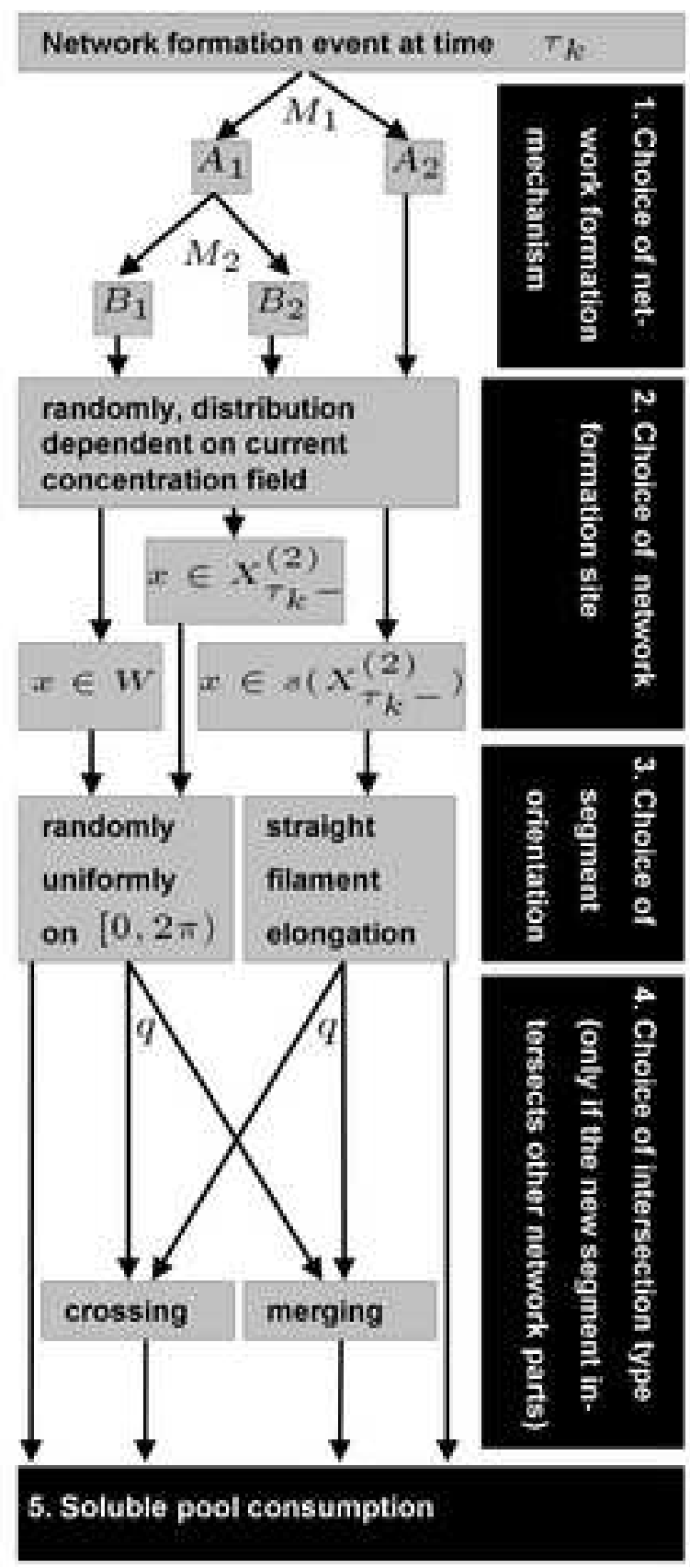

Figure 2: Illustration of model behavior at a network formation time $\tau_{k}$. Definitions: $A_{1}$ initiation of a new filament; $A_{2}$ elongation of a filament: $B_{1}$ free nucleation of a filament; $B_{2}$ lateral annealing: $x$ location in the observation window $W ; X_{\tau_{-}}^{(2)}$ and $s\left(X_{\tau_{k}-}^{(2)}\right)$ network and filament ends before the formation event, respectively: $M_{1}, M_{2}, q$ model parameters determining the probabilities of filament initiation vs. elongation, nucleation vs. lateral annealing and merging of a filament end with other network parts vs. crossing, respectively. 


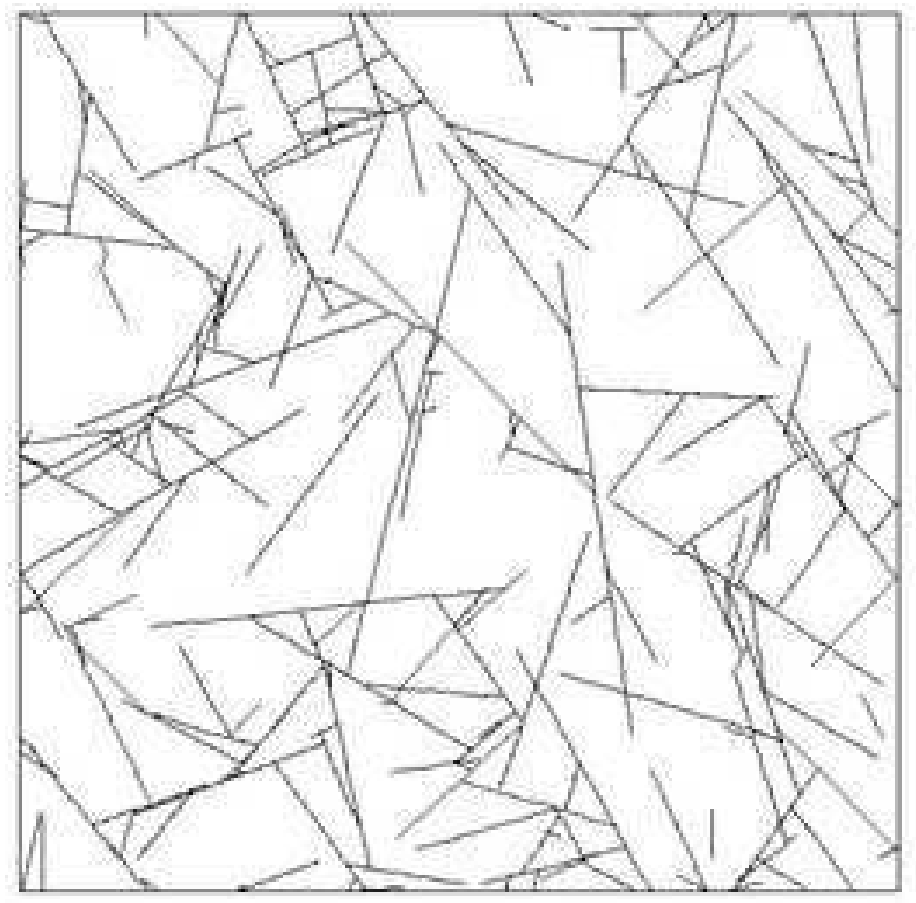

$$
c=2000
$$

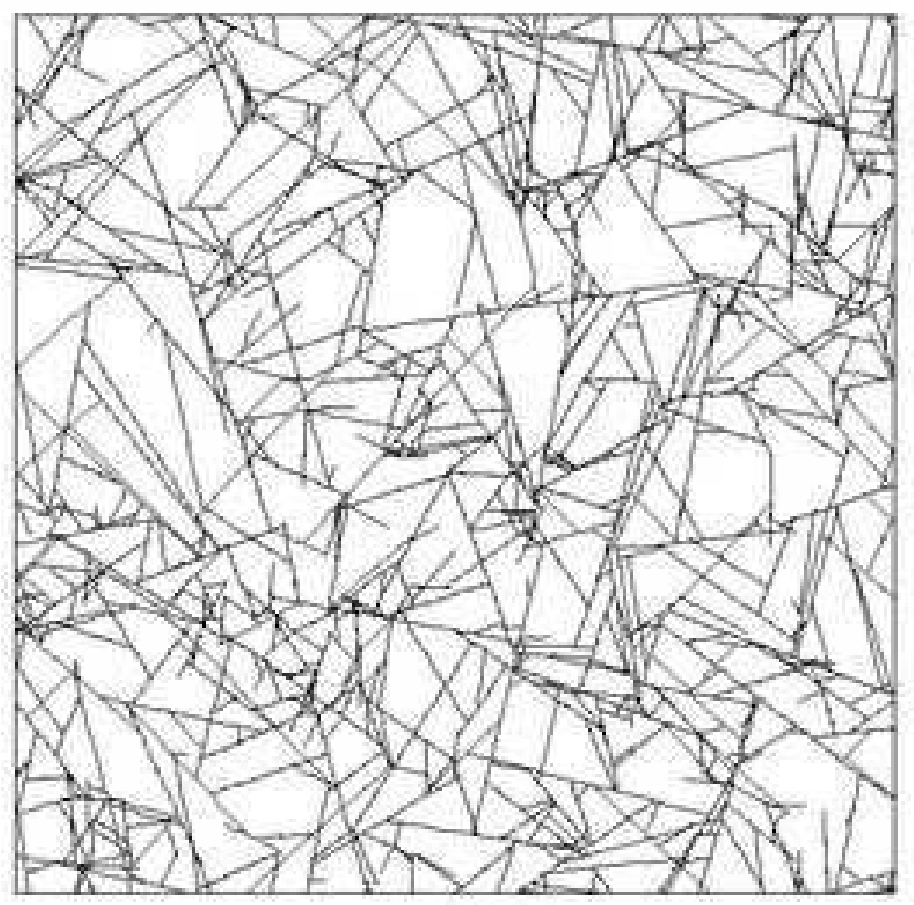

$$
c=5000
$$

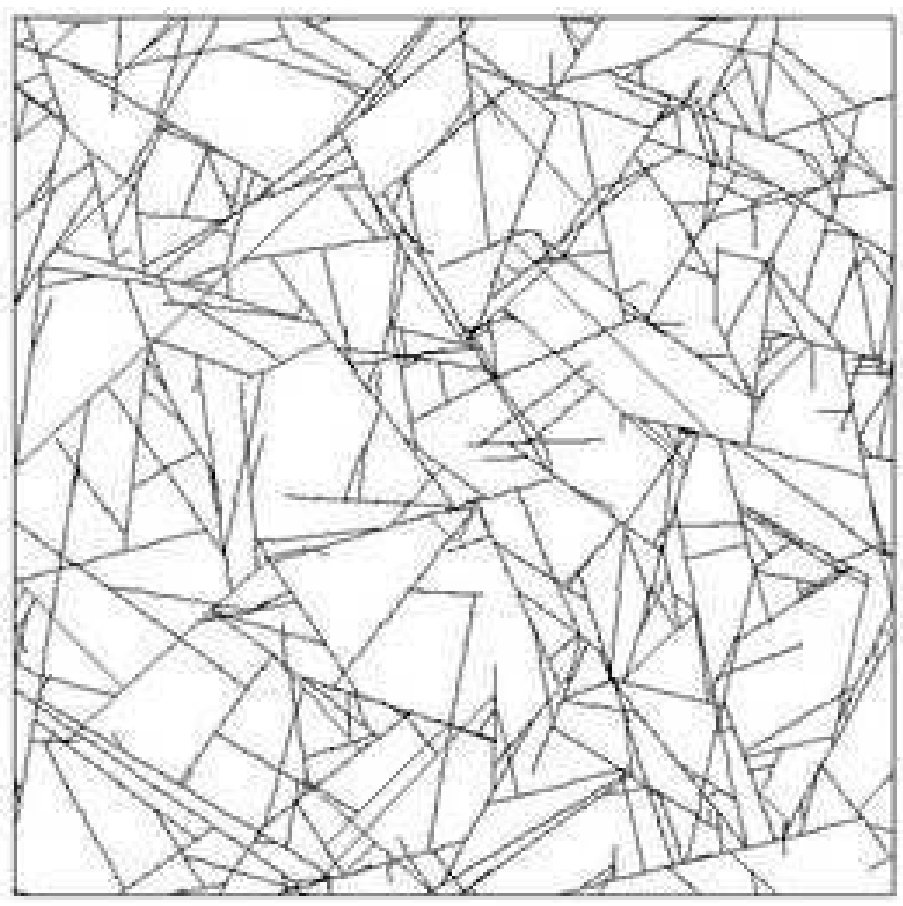

$$
c=3500
$$

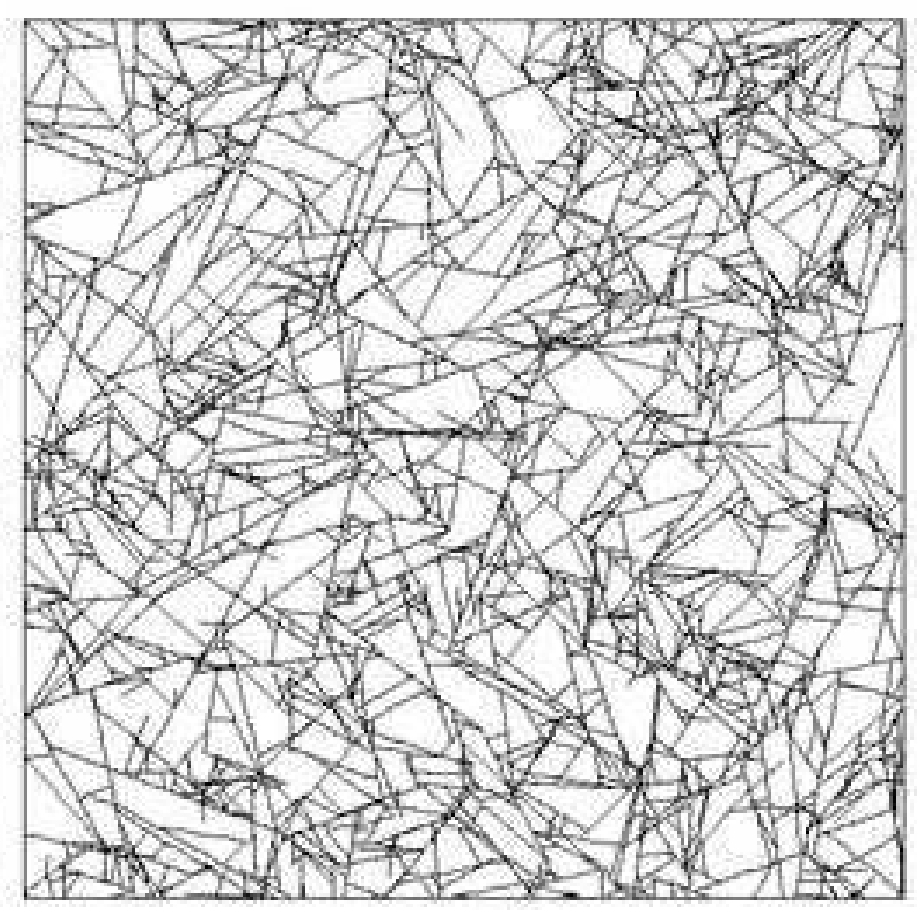

$$
c=7500
$$

Figure 3: Sample simulation outputs for different initial concentrations $c$ of soluble keratins. 


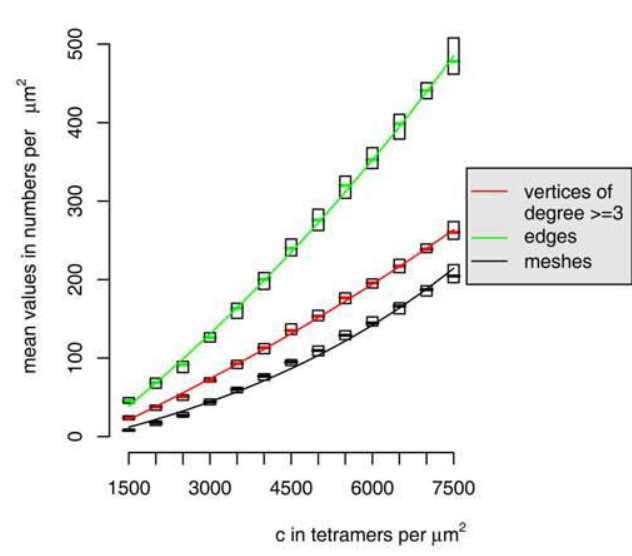

A

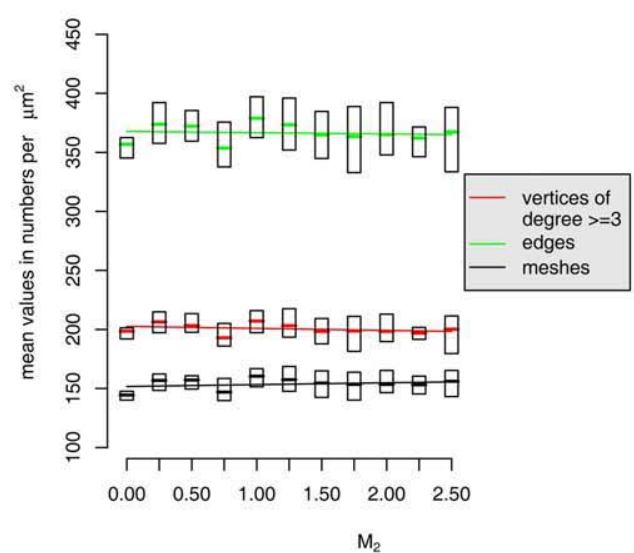

C

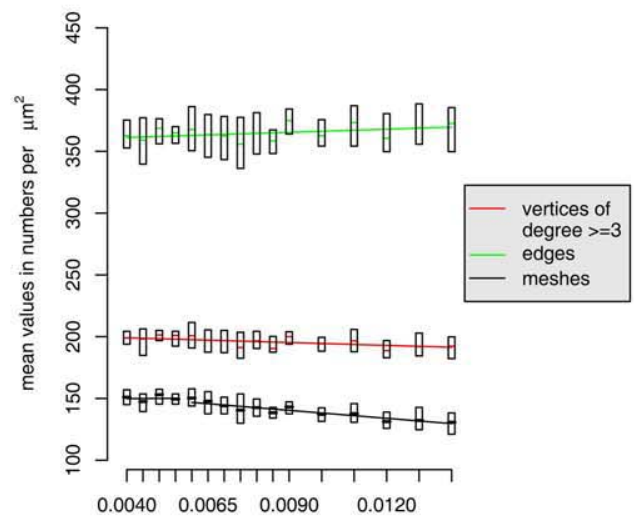

$M_{1}$

E

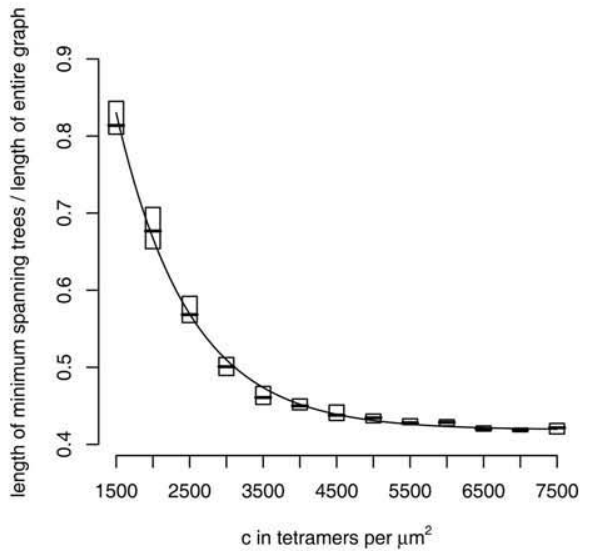

B

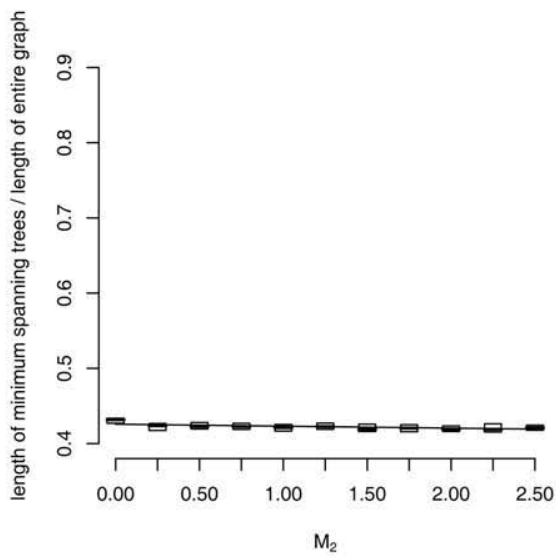

D

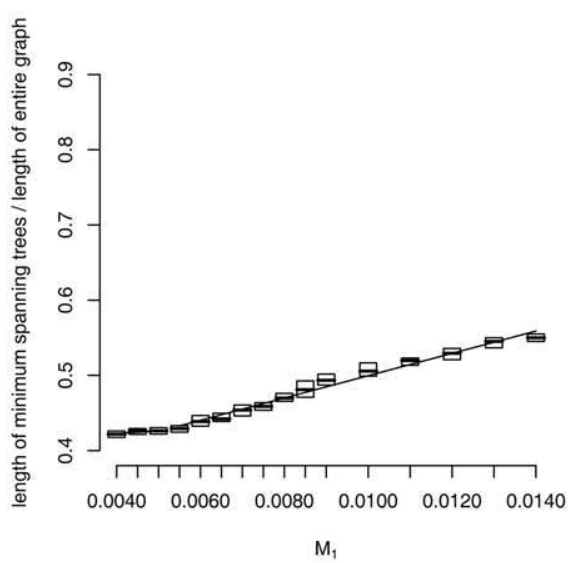

$\mathbf{F}$

Figure 4: A, B Response of the network morphology to variations of $c$ in tetramers per $\mu m^{2}$. The mean number of vertices of degree more than 3 , the mean number of edges and the mean number of meshes per $\mu \mathrm{m}^{2}$ grew almost linearly in $c$. The exponential decay of the relative MST-length indicates that network connectivity increased in $c$ and showed a stabilizing tendency above a critical level of $c=3500$ tetramers per $\mu \mathrm{m}^{2}$. C, D Response of the mean value characteristics to variations of the parameter $M_{2}$ controlling the likelihood of lateral keratin annealing vs. free nucleation. Neither the mean value characteristics nor the network connectivity were substantially affected by variations of $M_{2}$. E, F Response of the mean-value characteristics to variations of the parameter $M_{1}$ controlling the likelihood of filament initiation in comparison to elongation. For $M_{1} \geq 0.006$ there was a substantial decay of the mean number of meshes in $M_{1}$, whereas the other mean value characteristics were merely affected. The increasing relative MST-length indicated a loss of network connectivity if by increasing $M_{1}$ filament initiation was favored in comparison to filament elongation 


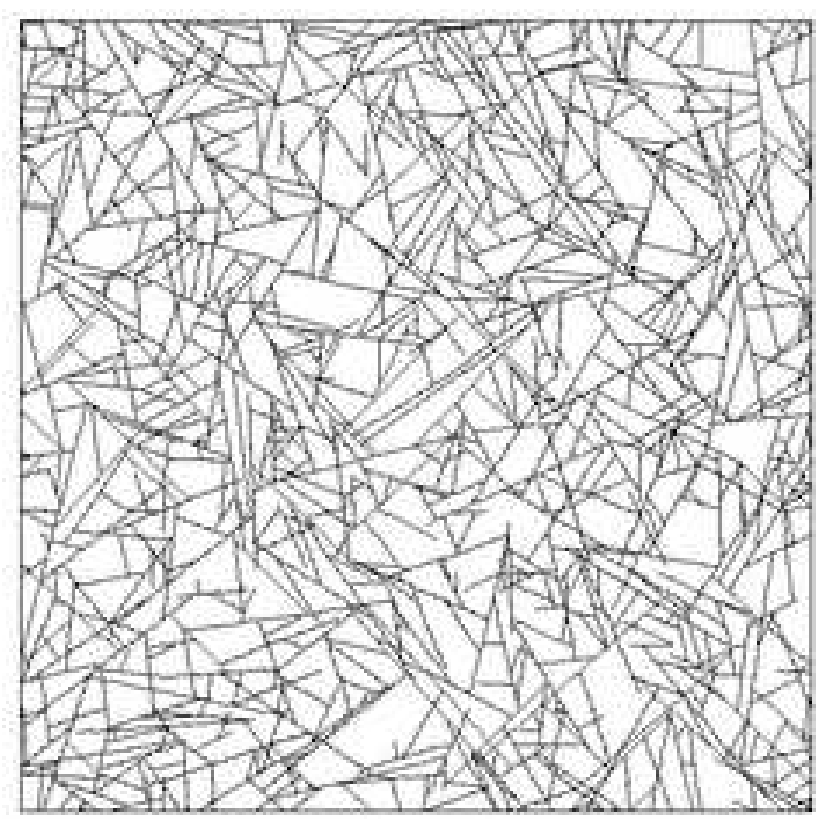

$$
M_{2}=0
$$

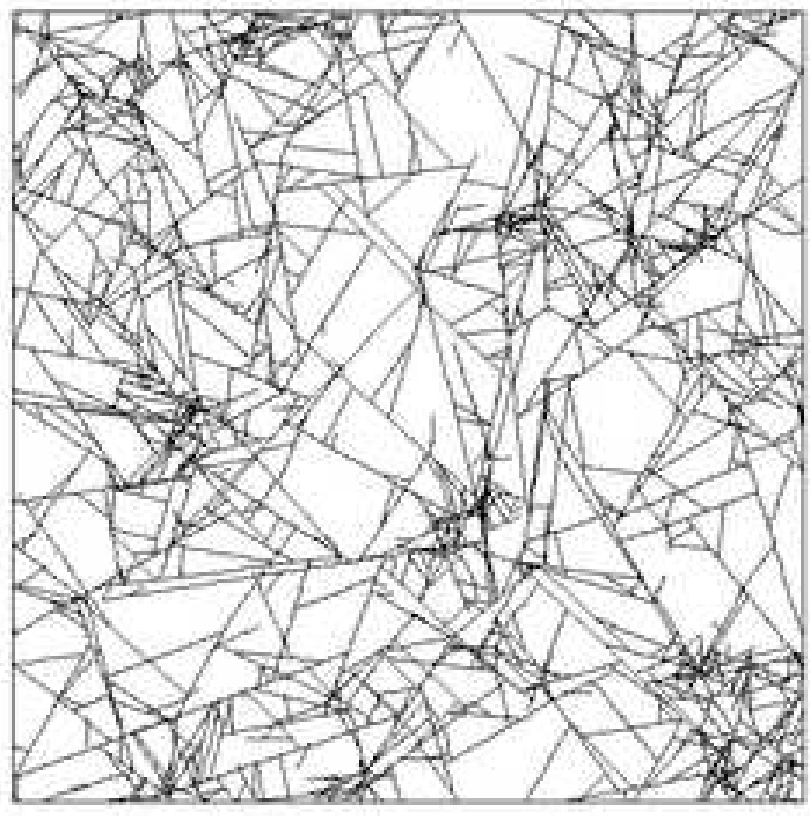

$$
M_{2}=1.5
$$

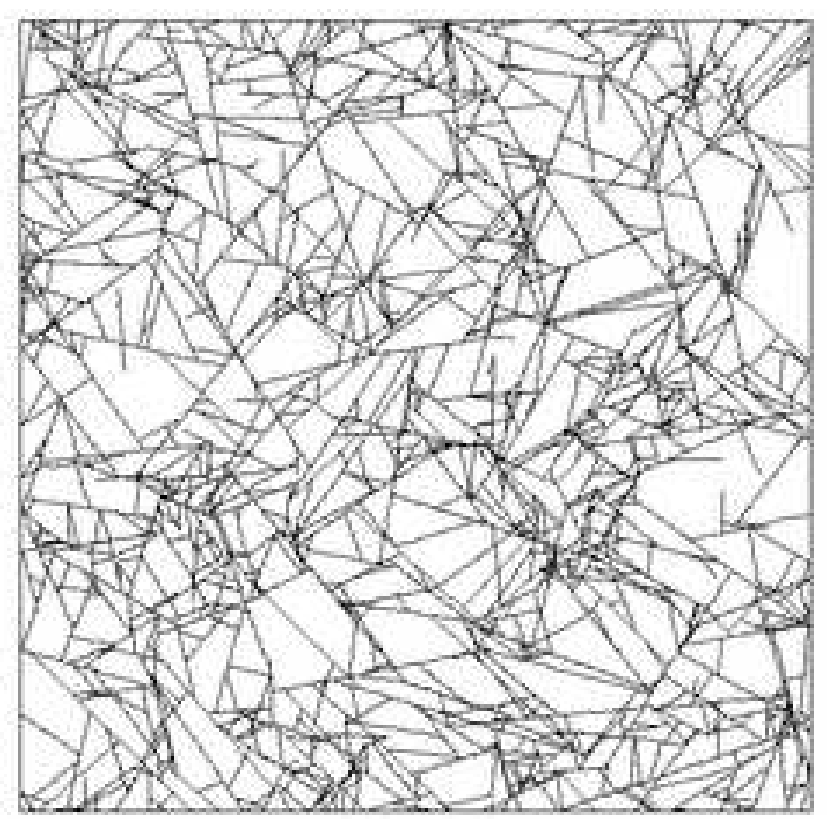

$$
M_{2}=0.25
$$

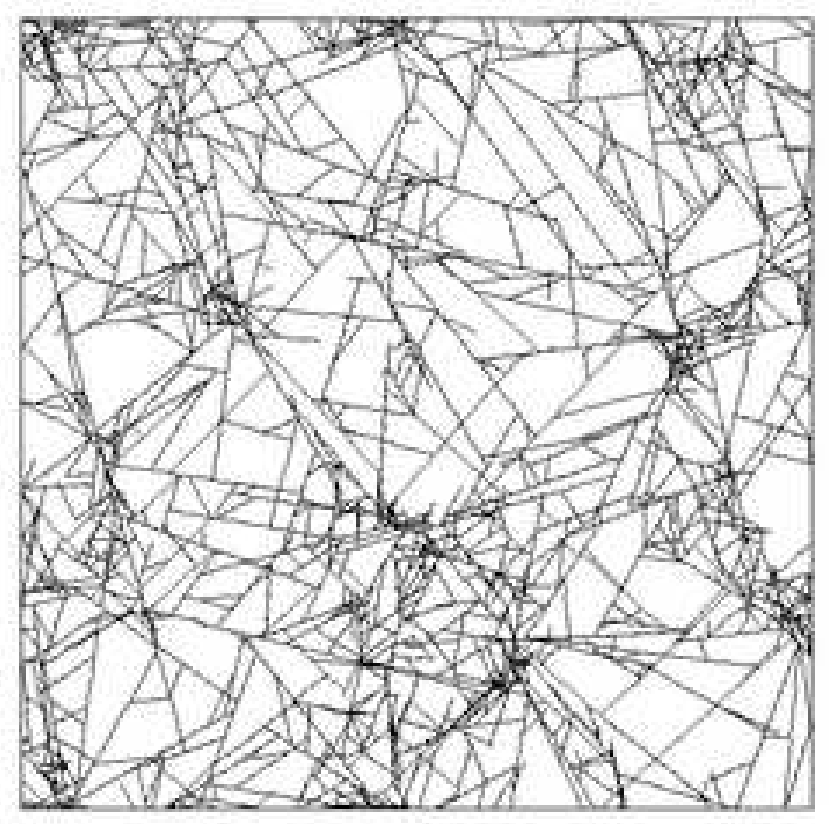

$$
M_{2}=2.0
$$

Figure 5: Sample simulation outputs for different values of $M_{2}$. It is visually noticeable that clustering effects were strengthened if $M_{2}$ was increased. 


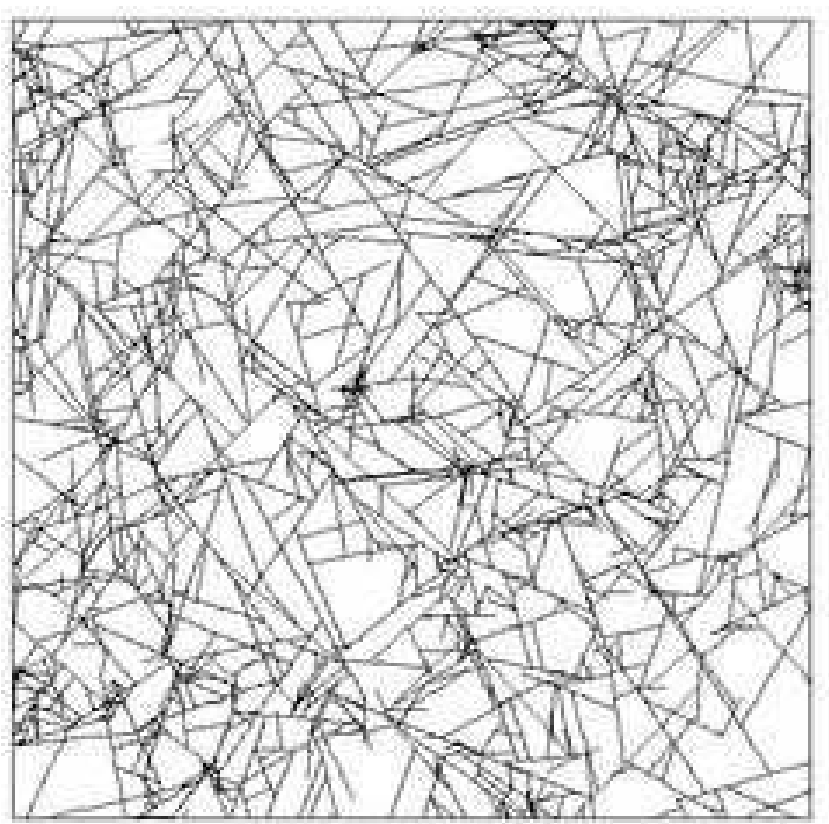

$$
M_{1}=0.004
$$

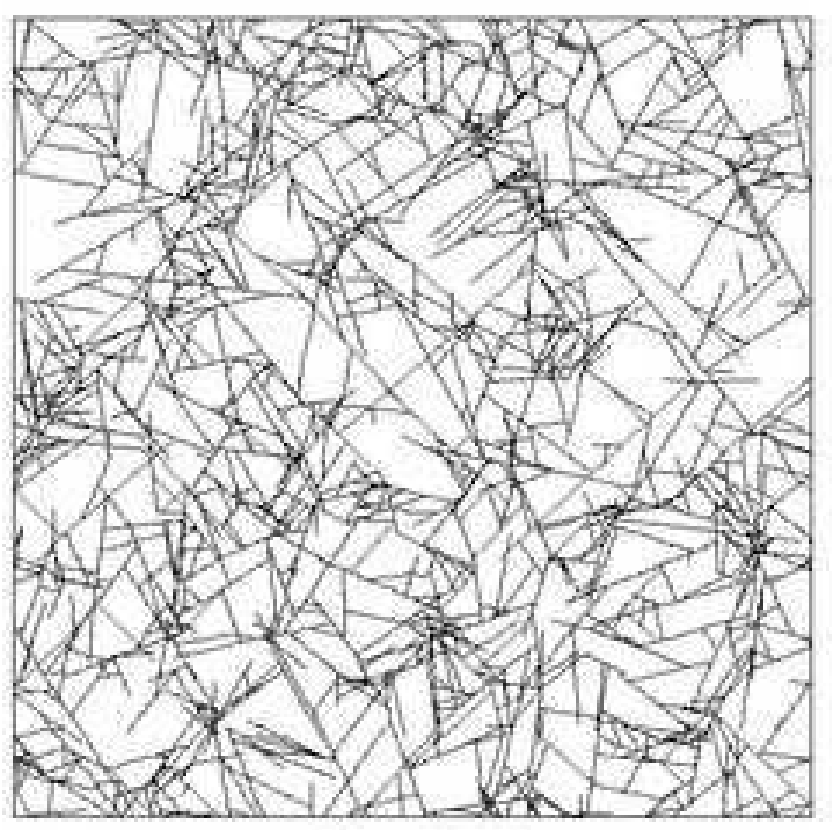

$$
M_{1}=0.008
$$

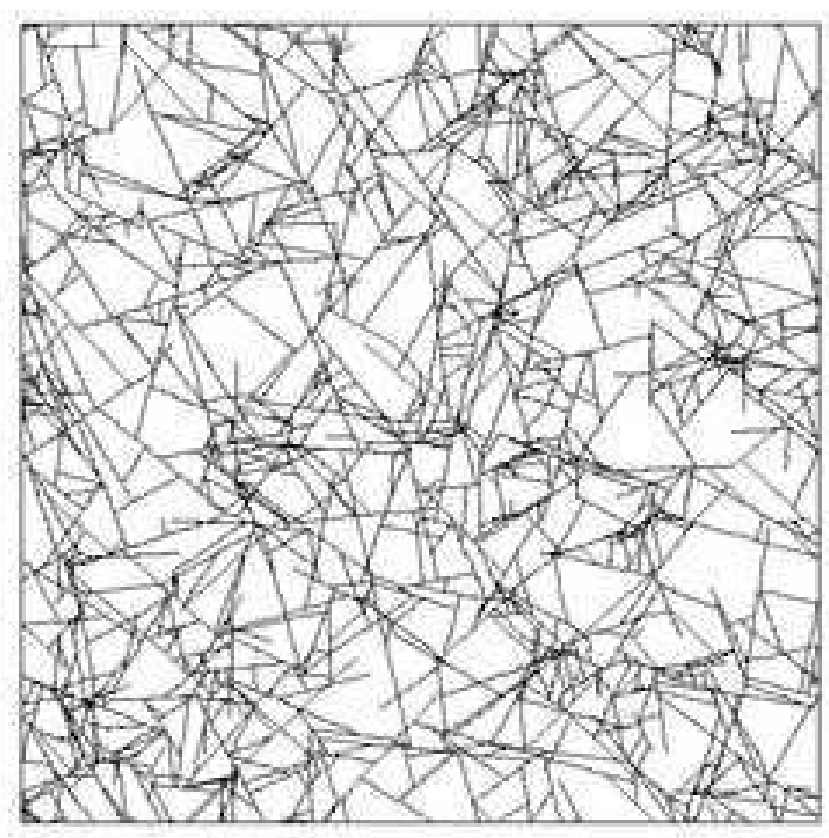

$$
M_{1}=0.006
$$

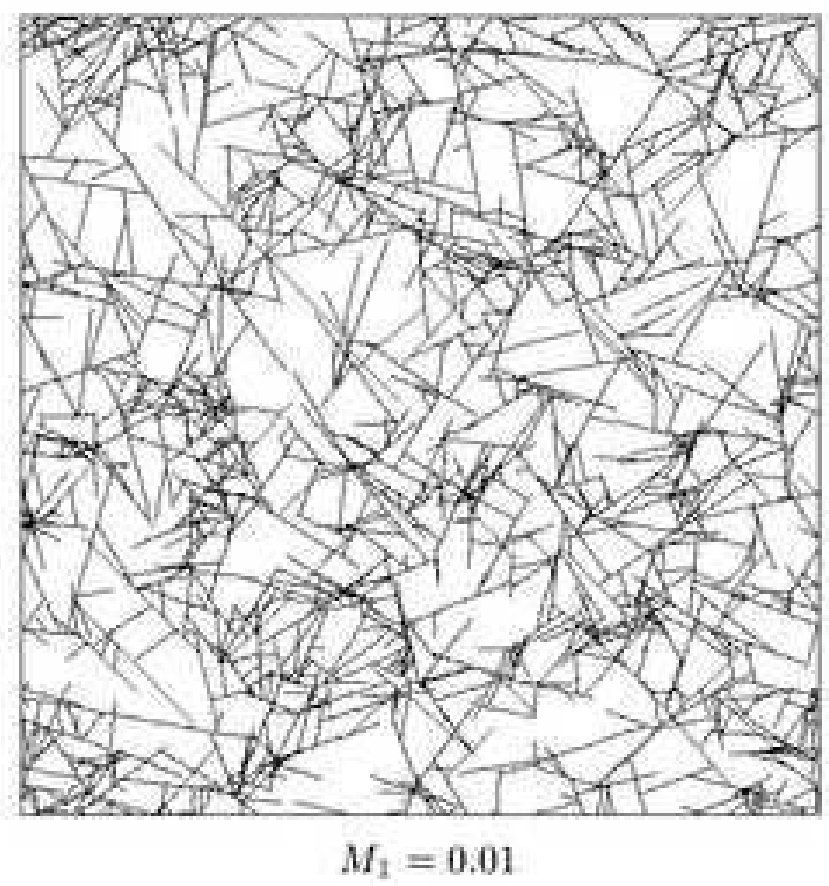

Figure 6: Sample simulation outputs for different values of $M_{1}$, It is visually noticeable that network connectivity decreased in $M_{1}$. 

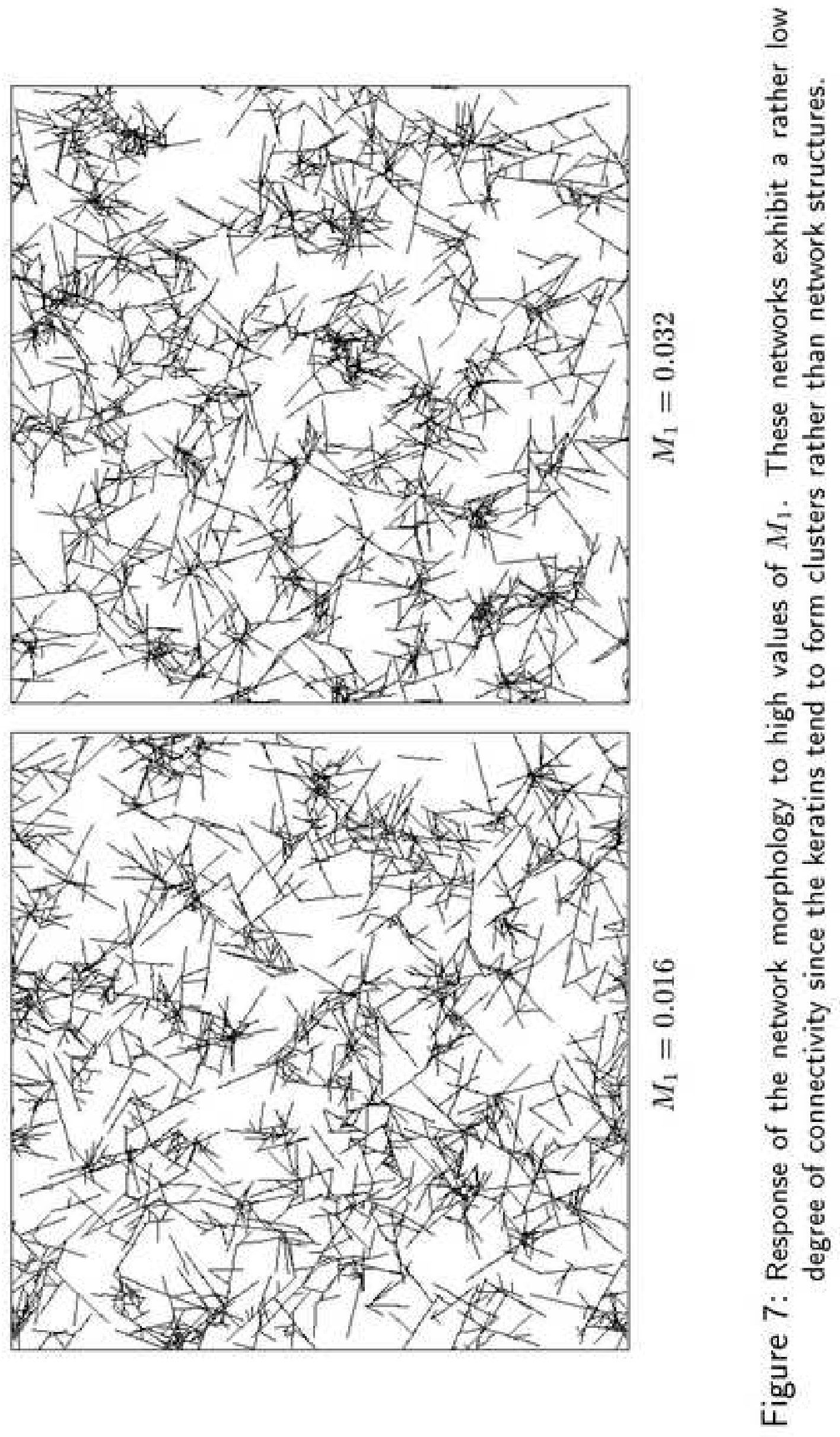

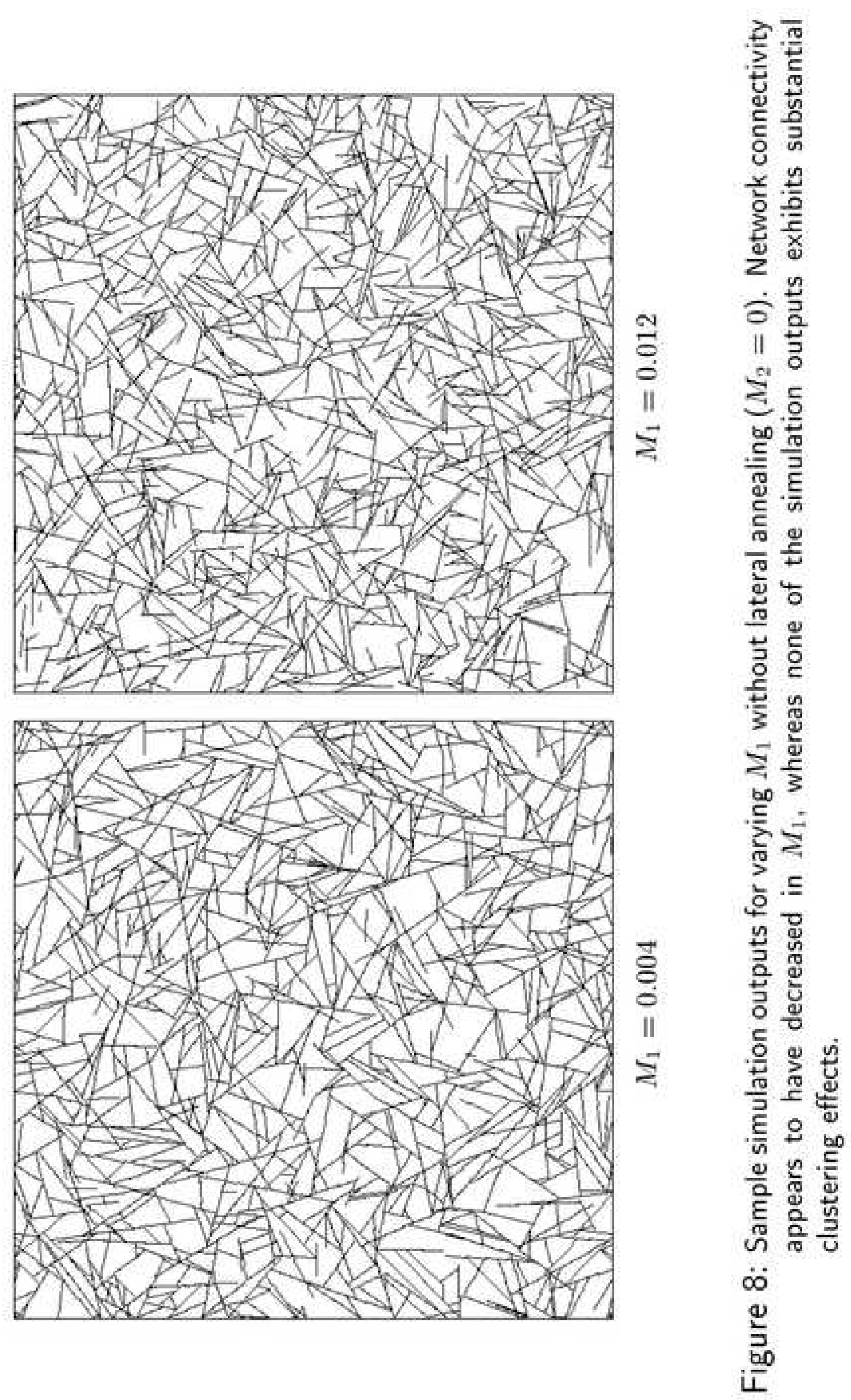


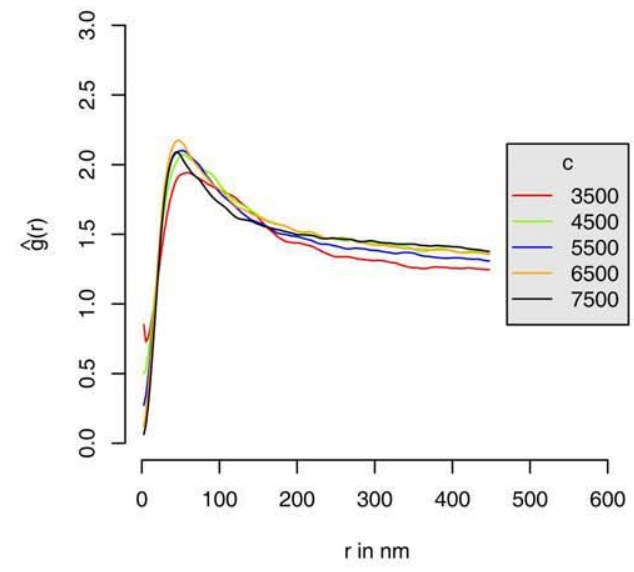

A Mesh clustering in response to variations of $c$ in tetramers $/ \mu m^{2}$

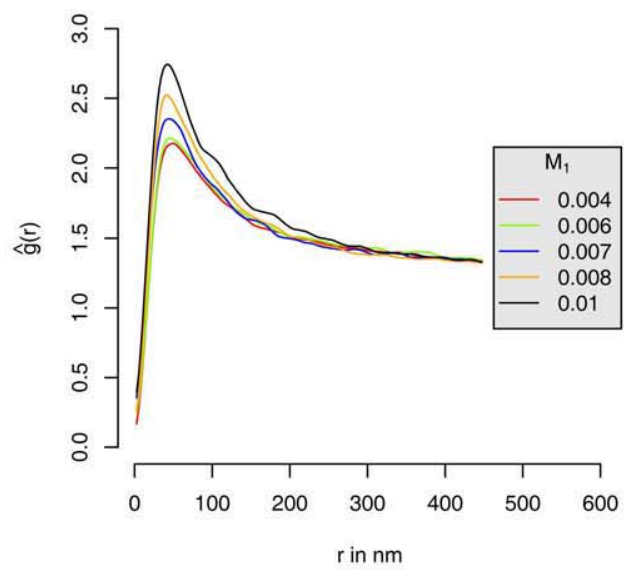

C Mesh clustering in response to $M_{1}$ with lateral D Mesh clustering in response to $M_{1}$ without lateral annealing $\left(M_{2}=0.22\right)$

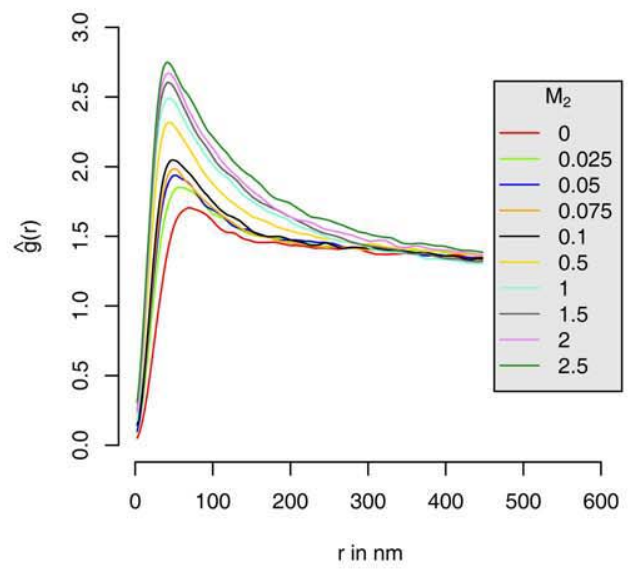

B Mesh clustering in response to $M_{2}$

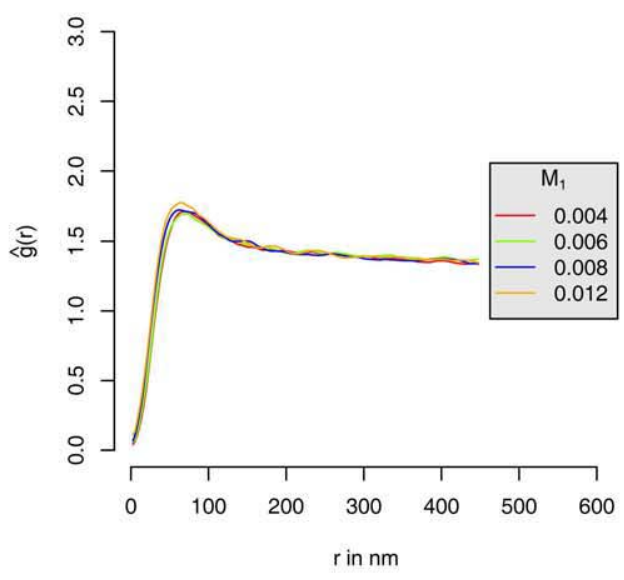

annealing $\left(M_{2}=0\right)$

Figure 9: A Clustering tendency of network meshes in response to variations of $c$. The pair correlation function was hardly affected by variations of $c$. B Clustering tendency of network meshes in response to variations of the parameter $M_{2}$ controlling the frequency of lateral annealing. The likelihood of mesh center distances between 50 and $150 \mathrm{~nm}$ increased in $M_{2}$ indicating the formation of mesh clusters. C and D Clustering tendency of meshes in response to the parameter $M_{1}$, controlling the likelihood of filament initiation in comparison to filament elongation. In the standard scenario, which included lateral keratin annealing, clustering effects increased in $M_{1}$. If lateral annealing was excluded $\left(M_{2}=0\right)$ and all new filaments were initiated by free nucleation, $M_{1}$ did not affect the pair correlation function. 


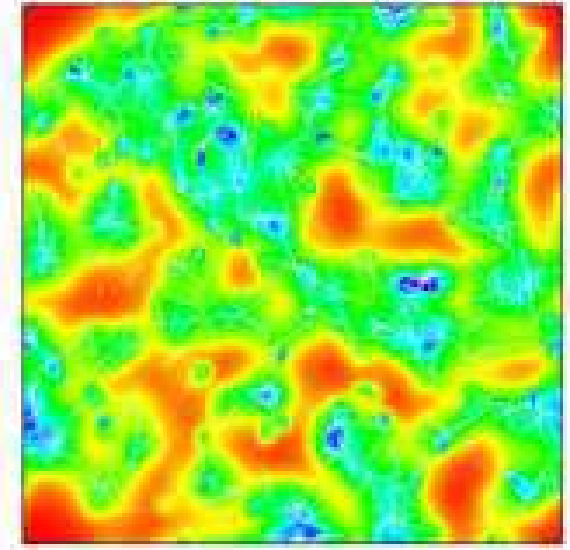

$D=5 \times 10^{-5} \mu m^{2} s^{-1}$

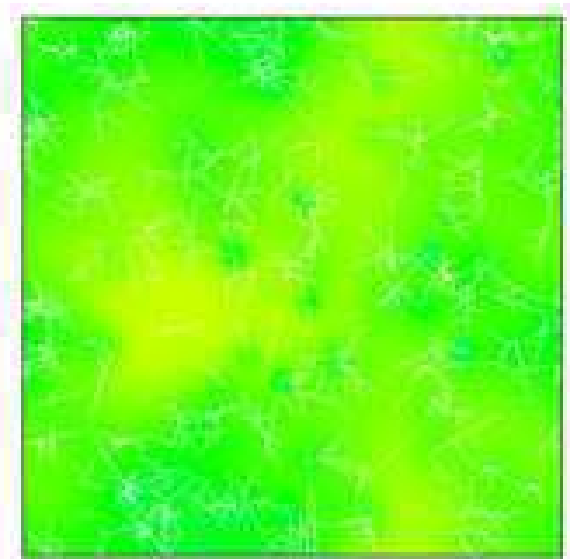

$D=8 \times 10^{-4} \mu m^{2} s^{-1}$

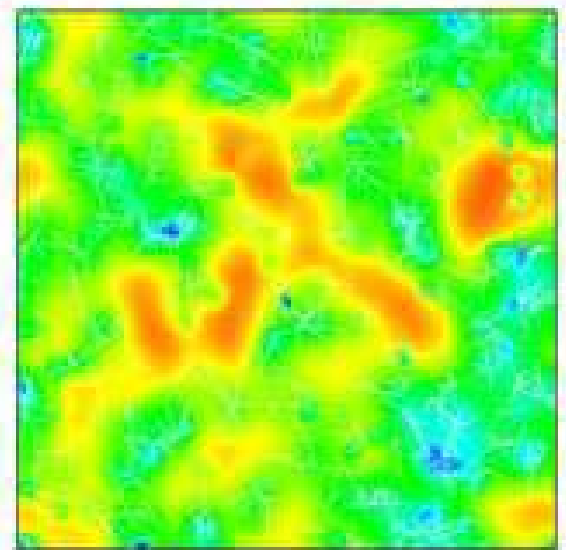

$D=1 \times 10^{-4} \mu m^{2} s^{-1}$

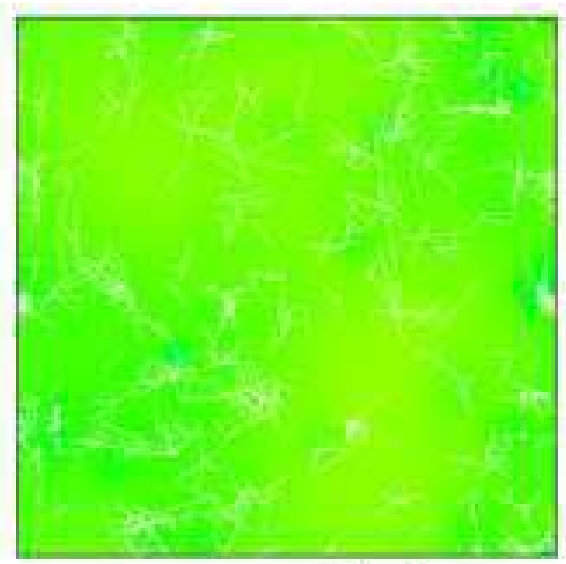

$D=3.2 \times 10^{-3} \mu m^{2} s^{-1}$

0

6200

Figure 10: Samples of concentration fields at time $t=120 \mathrm{~s}$ for different diffusion coefficients $D$. The parameter choices of $M_{1}=0.012$ and $M_{2}=0.22$ resulted in rapidly elongating filaments and the formation of mesh clusters and thus require relatively high diffusion coefficients in order to avoid local soluble pool depletion. For higher diffusion coefficients such as $D \geq 8 \times 10^{-4} \mu m^{2} s^{-1}$ depletion zones could not be observed. For small values of $D$ all depletion zones appeared to have only small diameters. 


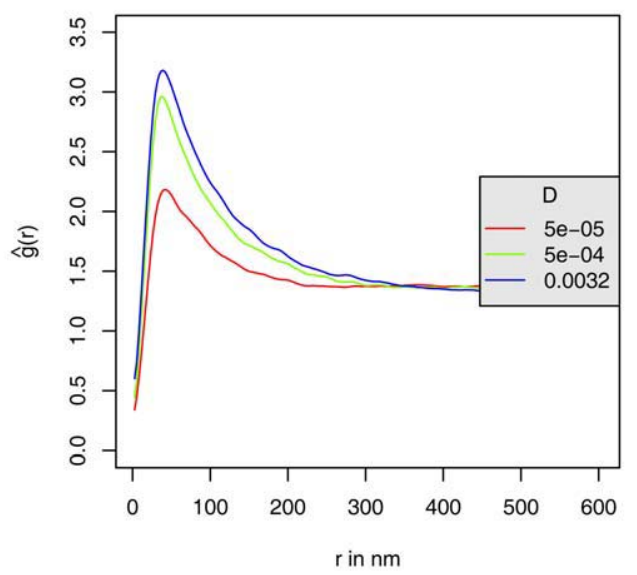

Figure 11: Mesh clustering in response to variations of the diffusion coefficient $D$. Very small diffusion coefficients interfered with the formation of mesh clusters, whereas mesh clustering was enhanced by rapid diffusion of filament precursor molecules. 

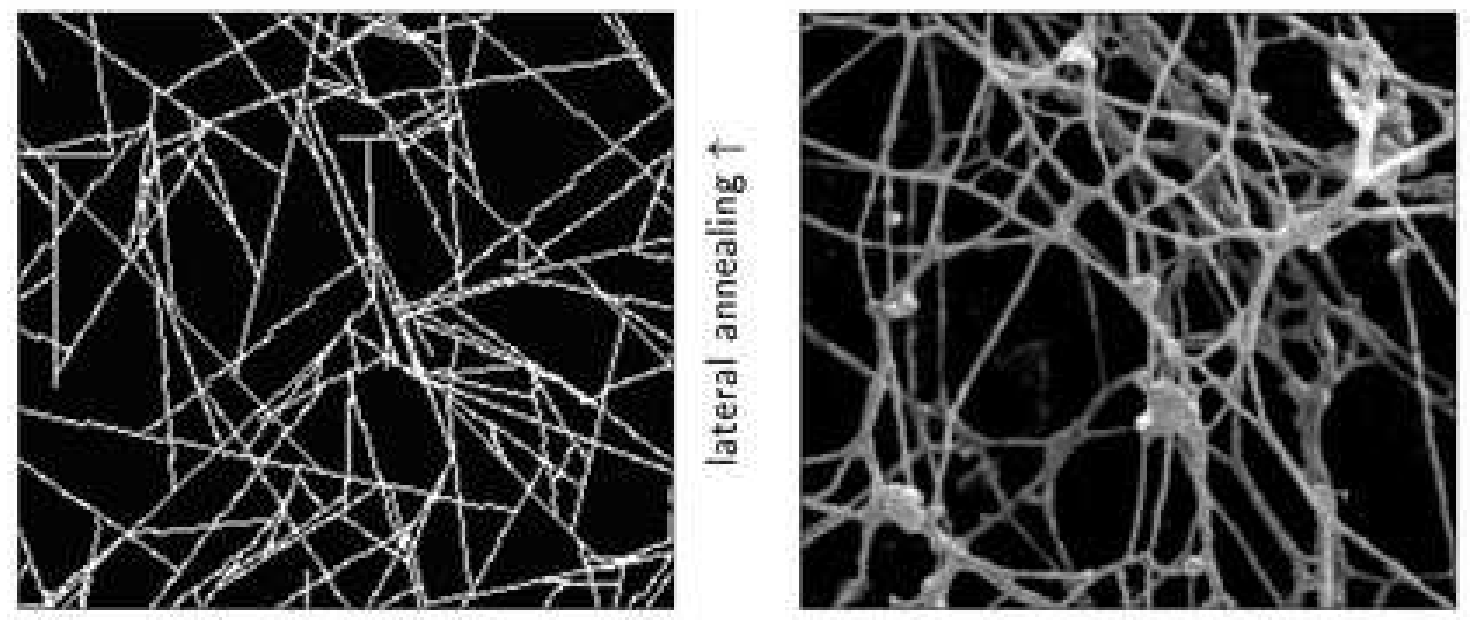

면

谆冠
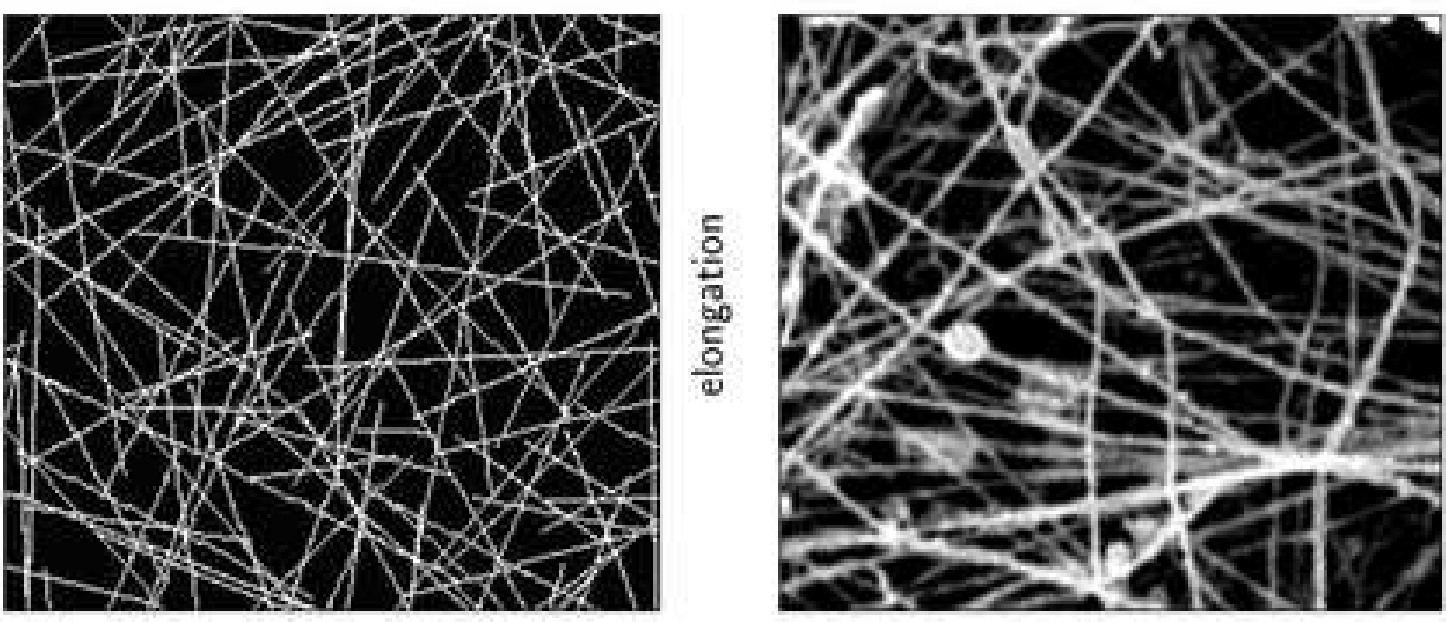

荧

$x$ E

要㐫

证

突

紊皆

ริํㅗ

힌

을

ที

引

힏으

들 흥

ษั 온

पू है

들 등

E 는

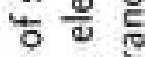

드

步

总导焉

ஸे

䅧 